\title{
The catalytic region and PEST domain of PTPN18 distinctly regulate the HER2 phosphorylation and ubiquitination barcodes
}

Hong-Mei Wang ${ }^{1,2, *}$, Yun-Fei Xu ${ }^{3,2, *}$, Shang-Lei Ning ${ }^{3, *}$, Du-Xiao Yang ${ }^{1}, \mathrm{Yi} \mathrm{Li}^{4}$, Yu-Jie Du ${ }^{2}$, Fan Yang ${ }^{2}$, Ya Zhang ${ }^{2}$, Nan Liang ${ }^{1,4}$, Wei Yao ${ }^{2}$, Ling-Li Zhang ${ }^{1}$, Li-Chuan Gu${ }^{5}$, Cheng-Jiang Gao ${ }^{1}$, Qi Pang ${ }^{4}$, Yu-Xin Chen ${ }^{3}$, Kun-Hong Xiao ${ }^{6}$, Rong $\mathrm{Ma}^{3}$, Xiao Yu${ }^{1,2,3}$, Jin-Peng Sun ${ }^{1,4}$

${ }^{I}$ Key Laboratory for Experimental Teratology of the Ministry of Education and Department of Biochemistry and Molecular Biology, Shandong University School of Medicine, Jinan, Shandong 250012, China; ${ }^{2}$ Department of Physiology, Shandong University School of Medicine, Jinan, Shandong 250012, China; ${ }^{3}$ Qilu Hospital, Shandong University, Jinan, Shandong 250012, China; ${ }^{4}$ Shandong Provincial Hospital, Shandong University, Jinan, Shandong 250021, China; ${ }^{5}$ Shandong University, School of Life Science, Jinan, Shandong 250012, China; ${ }^{6}$ Duke University, School of Medicine, Durham, 27705, USA

The tyrosine phosphorylation barcode encoded in C-terminus of HER2 and its ubiquitination regulate diverse HER2 functions. PTPN18 was reported as a HER2 phosphatase; however, the exact mechanism by which it defines HER2 signaling is not fully understood. Here, we demonstrate that PTPN18 regulates HER2-mediated cellular functions through defining both its phosphorylation and ubiquitination barcodes. Enzymologic characterization and three crystal structures of PTPN18 in complex with HER2 phospho-peptides revealed the molecular basis for the recognition between PTPN18 and specific HER2 phosphorylation sites, which assumes two distinct conformations. Unique structural properties of PTPN18 contribute to the regulation of sub-cellular phosphorylation networks downstream of HER2, which are required for inhibition of HER2-mediated cell growth and migration. Whereas the catalytic domain of PTPN18 blocks lysosomal routing and delays the degradation of HER2 by dephosphorylation of HER2 on $\mathrm{pY}^{1112}$, the PEST domain of PTPN18 promotes K48-linked HER2 ubiquitination and its rapid destruction via the proteasome pathway and an HER2 negative feedback loop. In agreement with the negative regulatory role of PTPN18 in HER2 signaling, the HER2/PTPN18 ratio was correlated with breast cancer stage. Taken together, our study presents a structural basis for selective HER2 dephosphorylation, a previously uncharacterized mechanism for HER2 degradation and a novel function for the PTPN18 PEST domain. The new regulatory role of the PEST domain in the ubiquitination pathway will broaden our understanding of the functions of other important PEST domain-containing phosphatases, such as LYP and PTPN12.

Keywords: phosphatase; receptor; ubiquitination; HER2; PTPN18; EGFR

Cell Research (2014) 24:1067-1090. doi:10.1038/cr.2014.99; published online 1 August 2014

*These three authors contributed equally to this work.

Correspondence: Xiao Yua ${ }^{\mathrm{a}}$, Jin-Peng Sun ${ }^{\mathrm{b}}$

åE-mail: yuxiao@sdu.edu.cn

${ }^{b}$ E-mail: sunjinpeng@sdu.edu.cn

Received 12 January 2014; revised 27 April 2014; accepted 26 May 2014; published online 1 August 2014

Abbreviations: AKT (protein kinase B); BDP1 (brain-derived phosphatase); c-Cbl (casitas B-lineage lymphoma proto-oncogene); DOK-R (Dok-related docking protein); EGF (epidermal growth factor); EGFR (epidermal growth factor receptor); ERK (extracellular signal-regulated kinase); Gab1 (Grb2-associated binder 1); GRB2 (growth factor receptor bound protein 2); HEPTP (hematopoietic protein tyrosine phosphatase); HER2 (human epidermal growth factor receptor-2); IGF1R (insulin-like growth factor 1 receptor); MAPK (mitogen-activated protein kinases); PI3K (phosphatidylinositol 3-kinases); PTP (protein tyrosine phosphatase); PTyr/pY (Phospho-tyrosine); RAS (rat sarcoma viral oncogene); SRC (sarcoma viral oncogene); CTH (C-terminal homology domain); STAT3 (signal transducer and activator of transcription 3); TCPTP (T-cell PTP) 


\section{Introduction}

Inappropriate responses to growth factors drive abnormal cell growth and transformation, which play crucial roles in the development and progression of human cancer [1-4]. Research on the action of growth factors led to the discovery of tyrosine phosphorylation and the receptor tyrosine kinase family in the early 1980s, including the receptors for epidermal growth factor (EGF) and insulin [5-8]. Cloning of EGF-activated receptors also identified the human EGF receptor (EGFR)-related gene 2, called HER2 or ErbB2. HER2 currently attracts extensive attention because its overexpression indicates poor prognosis in mammary, ovarian, gastric and other cancers $[9,10]$. Continuous endeavors focused on HER2 have resulted in the first targeted anti-tyrosine kinase therapy, Trastuzumab, for the treatment of metastatic breast and ovarian cancers, which has achieved remarkable success [10].

HER2 is normally regarded as an orphan receptor. Upon the action of growth factors, HER2 is the preferred dimerization partner of other EGFR family members in initiating downstream cellular signaling. The heterodimerization of HER 2 promotes an asymmetric interaction between the two kinase domains of each EGFR member, leading to elevated kinase activity and the specific tyrosine phosphorylation and ubiquitination of the HER2 C-terminal cytoplasmic domain [11] (Figure 1A). Recent studies have demonstrated that the HER2 C-terminal phosphorylation and ubiquitination encode important cellular functions, such as cell survival, proliferation, polarity, migration and protein degradation [12-16].

Among the six known HER2 C-terminal tyrosine phosphorylation sites, the phosphorylation of the juxtaposed tyrosine $\mathrm{Y}^{1221}$ and $\mathrm{Y}^{1222}$ enables high affinity for SHC1 and PIK3R3 binding and is a predictor of poor survival rates in breast cancer patients $[12,13,17]$. The phosphorylation of another HER2 residue, $\mathrm{Y}^{1248}$, recruits DOK-R, activates MAPK signaling and correlates with invasive ductal carcinomas or breast cancer in females $[16,18,19]$. Whereas the phosphorylation of $\mathrm{Y}^{1139}$ increases STAT3 transcriptional activity and activates RAS through GRB2 [14, 20], the phosphorylation of $\mathrm{Y}^{1196}$ increases the binding affinity of HER2 to CRK, activating ERK through a RAS-independent pathway, which is required for HER2 to drive cell motility, disrupt apical-basal polarity and inhibit apoptosis [12, 13, 15]. In addition to these sites, the phosphorylation of HER2 $\mathrm{Y}^{1112}$ near the kinase domain plays an important role in regulation of HER2 ubiquitination through its interaction with c-Cbl E3 ligase [21]. As an orphan receptor, HER2 is less strongly regulated by degradation than EGFR after
EGF stimulation; however, the Cbl-dependent lysosome routing of HER2 is significantly increased by anti-HER2 antibody treatment [21]. In addition, Hsp90 inhibitory antibodies promote HER2 ubiquitination and proteasome targeting [22]. The precise HER2 C-terminal phosphorylation barcode and other mechanisms that promote HER2 ubiquitination are not fully understood.

The HER2 C-terminal tyrosine phosphorylation barcodes are precisely regulated by the concerted actions of both kinases and phosphatases. Recent research has identified several protein tyrosine phosphatases (PTPs), including PTP-MEG2, PTPN12, PTPN13 and PTPN18, involved in HER2 signaling in specific cellular contexts; however, the mechanism by which these phosphatases specifically control HER 2 tyrosine phosphorylation has never been explored in detail [23-26]. Compromised PTPN12 function is associated with triple-negative breast cancer, and PTPN13 mutations were found in a PIK3CA-targeted gene screen $[24,26]$. Therefore, in-depth knowledge of the precise role of each PTP in HER2 regulation is necessary if we try to understand HER2 signaling and develop more efficient HER2-targeted therapies.

PTPN18, also called BDP1, was the first HER2 tyrosine phosphatase identified [23]. Intriguingly, PTPN18 only dephosphorylates the phospho-tyrosine residues of HER2, not those of EGFR or IGF1R, suggesting the stringent substrate specificity of PTPN18 [23]. Structurally, PTPN18 belongs to the small sub-family of PEST domain-containing PTPs, which also includes LYP/ PTPN22 and PTP-PEST/PTPN12 [27-29]. In general, PTP substrate specificity is defined by a combination of selectivity in the catalytic region and the adjacent regulatory domains. Whereas the C-terminal regulatory domain is important for proper LYP function, our recent studies on LYP have revealed that unique structural characteristics in its $\mathrm{N}$-terminal catalytic region, including an LYP-specific insert and an adjacent serine-phosphorylation site, determine substrate specificity $[28,30]$. Similar to LYP, PTPN18 displays very low activity towards small artificial substrates, suggesting that a large structural rearrangement may occur upon the formation of the enzyme-substrate complex. To understand the mechanism underlying HER2 tyrosine phosphorylation and the substrate specificity of PTPN18, we set out to elucidate the action of PTPN18 on HER2 tyrosine phosphorylation by combined cellular, kinetic and crystallographic analyses. We have defined the structural basis and functional roles of catalytic region and PEST domain of PTPN18 in specific regulation of HER2 phosphorylation barcodes. Moreover, whereas the interaction of HER2 $\mathrm{pY}^{1196}$ and $\mathrm{pY}^{1248}$ with PTPN18 governs HER2-mediated cell migration and proliferation, we observed that the PTPN18 
A

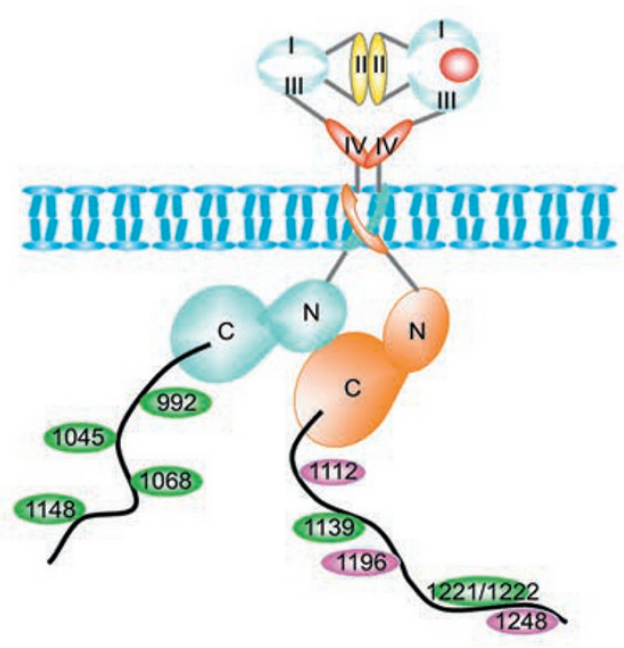

B

EGF

TIME

IB:p-HER2(1112)

IB:p-HER2(1139)

IB:p-HER2(1196)

IB:p-HER2(1221/1222)

IB:p-HER2(1248)

IB:Total-HER2

IB:p-EGFR(992)

IB:p-EGFR(1045)

IB:p-EGFR(1068)

IB:p-EGFR(1148)

IB:Total-EGFR

IB:PTPN18

IB: $\beta$-ACTIN
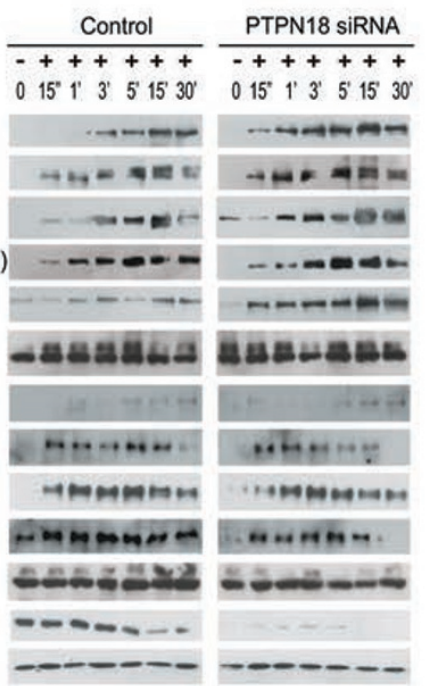

C

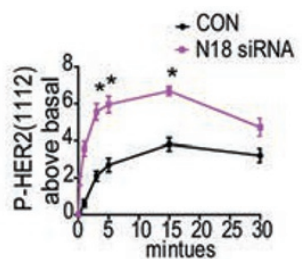

(1)

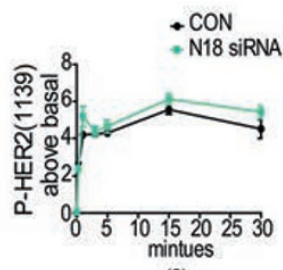

(2)

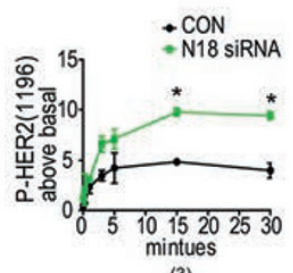

(3)

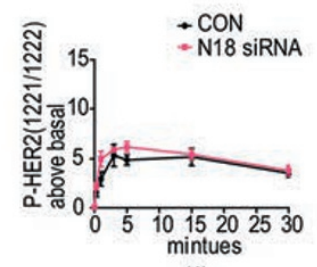

(4)

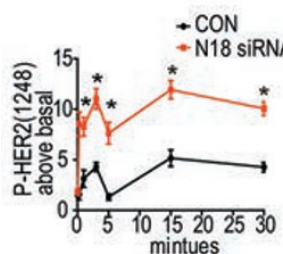

(5)

D

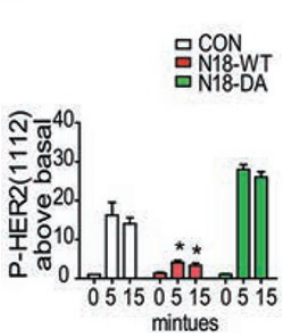

(1)

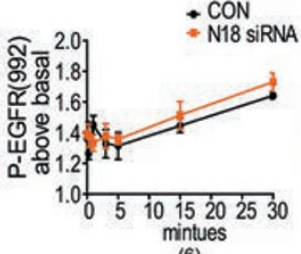

(6)

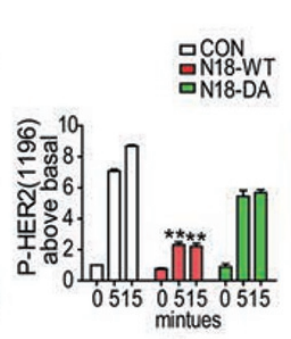

(2)

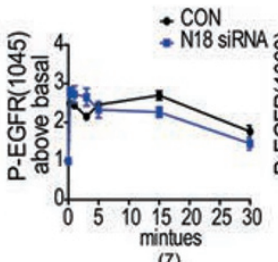

(7)

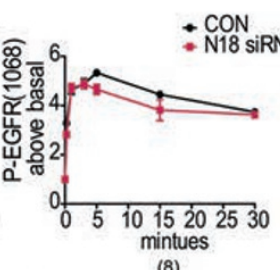

(8)

E

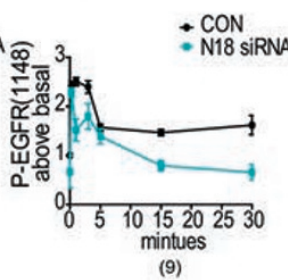

(9)

Figure 1 PTPN18 specifically regulates HER2 $Y^{1112}, Y^{1196}$ and $Y^{1248}$ phosphorylation states. (A) A model of HER2 activation via dimerization with EGFR and their C-terminal tyrosine phosphorylation sites. (B) PTPN18 knockdown selectively enhances the EGF-induced tyrosine phosphorylation of the HER2 $Y^{1112}, Y^{1196}$ and $Y^{1248}$ sites. (C) Tyrosine phosphorylation in B was quantified by densitometry and expressed as a ratio relative to the initial phosphorylation level. (D) Overexpression of PTPN18-WT specifically suppresses the EGF-induced phosphorylation of the HER2 $Y^{1112}, Y^{1196}$ and $Y^{1248}$ sites, whereas overexpression of an inactive PTPN18 mutant D197A has much less effect on the phosphorylation of the HER2 $Y^{1196}$ and $Y^{1248}$, and slightly increased the phosphorylation of HER2 $\mathrm{Y}^{1112}$. Tyrosine phosphorylation from Supplementary information, Figure S1A was quantified by densitometry and compared with the non-stimulated state. (E) Time course of dephosphorylation of immuno-purified phosphorylated HER2 by GST-PTPN18 in vitro. Flag-tagged HER2 was transiently transfected into HEK293 cells. After stimulation with $15 \mathrm{ng} / \mathrm{ml}$ EGF for 5 min to enrich HER2 phosphorylation, the cells were lysed and Flag-tagged-HER2 was purified with Flag-M2 affinity gel. HER2 dephosphorylation was monitored by western blotting with specific phospho-HER2 antibodies (Supplementary information, Figure S1B), and it was quantified by densitometry. All statistics shown represent the mean \pm SEM from at least three independent experiments. ${ }^{*} P<0.05,{ }^{* *} P<0.01$ compared with control. 
switches ubiquitination and degradation routes of HER2, which require both its catalytic region and $\mathrm{C}$-terminal PEST domain. The identification of the ancillary role of PTPN18 in mediating HER2 ubiquitination broadened our knowledge of the function of the PEST domain in protein phosphatases $[27,31]$.

\section{Results}

PTPN18 specifically regulates HER2 $Y^{1112}, Y^{1196}$ and $Y^{1248}$ phosphorylation states

The C-terminal domain of HER2 receptor contains a series of tyrosine phosphorylation sites (Figure 1A). To determine the specific sites regulated by PTPN18, we knocked down PTPN18 expression and monitored changes in the tyrosine phosphorylation of HER2 and EGFR (Figure 1B). In general, knockdown of PTPN18 by PTPN18-targeting siRNA did not perturb the phosphorylation states of the EGFR C-terminal $\mathrm{Y}^{992}, \mathrm{Y}^{1045}$, $\mathrm{pY}^{1068}, \mathrm{Y}^{1148}$ sites or the HER2 $\mathrm{Y}^{1139}$ and $\mathrm{Y}^{1221 / 1222}$ double sites (Figure 1B and 1C); however, the phosphorylation of the HER2 $\mathrm{Y}^{1112}, \mathrm{Y}^{1196}$ and $\mathrm{Y}^{1248}$ sites was significantly elevated in a time-dependent manner. To determine whether PTPN18 specifically regulate HER2 $\mathrm{pY}^{1112}$, $\mathrm{pY}^{1196}$ and $\mathrm{pY}^{1248}$ through its phosphatase activity, we overexpressed the wild-type PTPN18 (PTPN18-WT) and an inactive mutant (D197A) to evaluate their effects on EGF-induced HER2 phosphorylation. Overexpression of PTPN18-WT decreased the EGF-induced HER2 phosphorylation on $\mathrm{Y}^{1112}, \mathrm{Y}^{1196}$ and $\mathrm{Y}^{1248}$ by 3-4-fold, whereas the equal overexpression of the PTPN18-D197A did not significantly change the HER2 phosphorylation states of $\mathrm{Y}^{1196}$ and $\mathrm{Y}^{1248}$, but increased the phosphorylation of $\mathrm{Y}^{1112}$ (Figure 1D and Supplementary information, Figure S1A). To confirm the direct dephosphorylation of HER2 $\mathrm{pY}^{1112}, \mathrm{pY}^{1196}$ and $\mathrm{pY}^{1248}$ by PTPN18, we next immunoprecipitated phosphorylated HER2 after EGF stimulation and monitored the dephosphorylation of HER2 by GST-PTPN18 in vitro (Figure 1E and Supplementary information, Figure S1B). Whereas PTPN18 shows little activity toward the $\mathrm{pY}^{1139}$ or $\mathrm{pY}^{1221 / 1222}$ sites of immunoprecipitated HER2, it efficiently dephosphorylated immunoprecipitated HER2 at the $\mathrm{pY}^{1112}, \mathrm{pY}^{1196}$ and $\mathrm{pY}^{1248}$ sites. These results showed that PTPN18 specifically regulates HER2 $\mathrm{pY}^{1112}, \mathrm{pY}^{1196}$ and $\mathrm{pY}^{1248}$ upon EGF stimulation via its phosphatase activity.

PTPN18 specifically recognizes HER2 by synergistic actions of the catalytic region and PEST domain

The temporal regulation of the HER2 $\mathrm{Y}^{1112}, \mathrm{Y}^{1196}$ and $\mathrm{Y}^{1248}$ phosphorylation states by PTPN18 suggested the dynamic association between PTPN18 and HER2. There- fore, we overexpressed the PTPN18-WT and examined the receptor-phosphatase complexes by co-immunoprecipitation. The EGF-induced receptor-phosphatase complex formation was peaked at $15 \mathrm{~min}$ (Figure 2A and 2B). We next monitored the interaction of HER2 with the PTPN18 substrate trapping-mutant D197A. The D197 in PTPN18 functions as the general acid during catalysis and is required for the cleavage of the scissile P-O bond in the tyrosine phosphorylated substrate. Accordingly, the PTPN18-D197A trapping mutant bound to the substrate, but its dissociation rate for the substrate was significantly decreased. Stronger interactions between PTPN18 D197A and HER2 were detected, and the formation of the receptor-phosphatase complex was observed from the first minute (Figure 2A and 2B). The effect of the D197A mutation supports the "kiss and run" mechanism of interaction between HER2 and its phosphatase PTPN18. We next mapped the key elements of PTPN18 in mediating HER2 interaction by different PTPN18 truncations (Figure $2 \mathrm{C}$ and $2 \mathrm{D}$ and Supplementary information, Figure $\mathrm{S} 2 \mathrm{~A}$ ). Whereas the last 55 residues of the C-terminal PEST domain are required for the interaction of PTPN18-WT with HER2, the catalytic domain (CD) with the inactive C229S mutation also binds to HER2. Both $\mathrm{N}$ - and C-terminal interactions of PTPN18 with phosphorylated HER2 are specific and direct, as verified by an in vitro GST pull-down assay (Figure 2E). Therefore, whereas the C-terminal PEST domain of PTPN18 forms a relatively stable complex with HER2 after EGF stimulation, which required its last 55 residues, the $\mathrm{CD}$ of PTPN18 interacts with the phosphorylated tyrosine sites of HER2 transiently, with the "kiss and run" mechanism.

PTPN18 kinetically prefers HER2 $Y^{1112}, Y^{1196}$ and $Y^{1248}$ phosphorylation sites

PTPN18 specifically regulated three HER2 sites out of 10 HER2 and EGFR phosphorylation sites, probably through recognition of the local microenvironment of these sites, such as the primary peptide sequence. Therefore, synthetic phospho-peptides corresponding to the 13 known HER2 and EGFR tyrosine phosphorylation sites, together with five phospho-peptides derived from several proteins involved in downstream HER2 signaling, were assessed for in vitro PTPN18 catalytic activity. PTPN18 selectively recognized the HER2 $\mathrm{pY}^{1112}, \mathrm{pY}^{1196}$ and $\mathrm{pY}^{1248}$ phosopho-peptides over all other phospho-peptides (Figure $2 \mathrm{~F}$ and Supplementary information, Table S1). The $K_{\mathrm{m}}$ for PTPN18 catalysis of the HER2 $\mathrm{pY}^{1112}, \mathrm{pY}^{1196}$ and $\mathrm{pY}^{1248}$ phospho-peptides ranged from 6 to $23 \mu \mathrm{M}$, and $K_{\text {cat }}$ ranged from 4 to $11.2 \mathrm{~s}^{-1}$. The $K_{\text {cat }} / K_{\mathrm{m}}$ ratio, which primarily reflects the substrate association with the enzyme, is a good indicator of enzyme-substrate speci- 
A

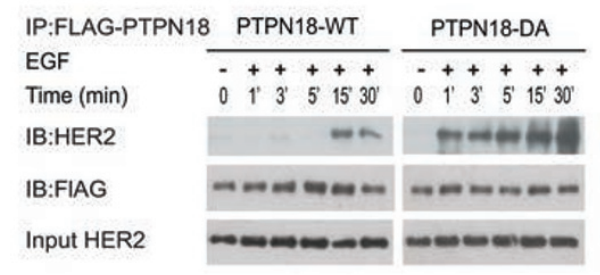

C

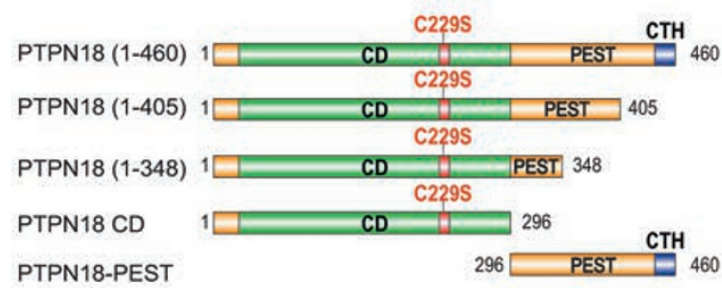

$\mathrm{E}$

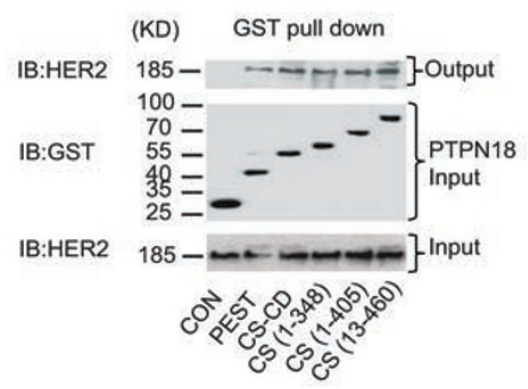

$\mathrm{F}$
B

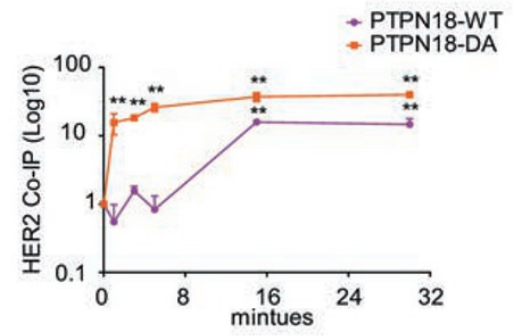

D

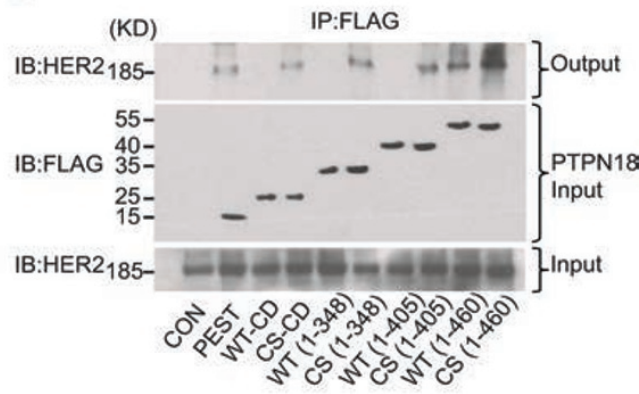

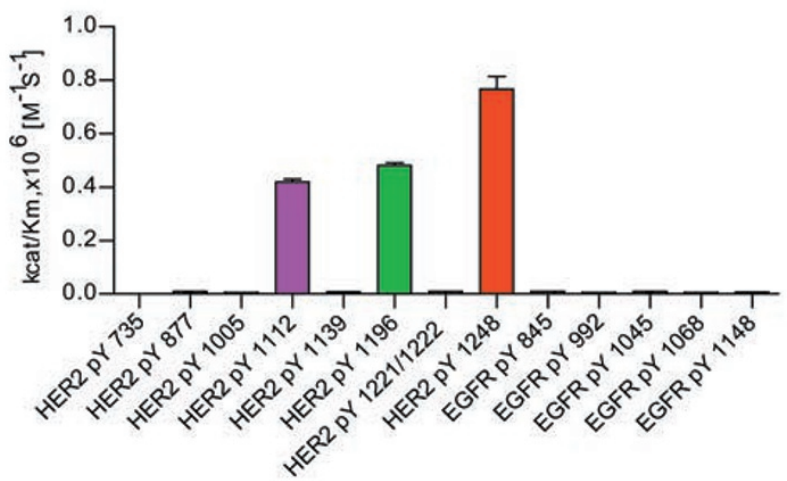

Figure 2 PTPN18 specifically recognizes HER2 by synergistic actions of the catalytic region and PEST domain. (A) EGF enhances the time-dependent PTPN18/HER2 complex formation. Equal amounts of FLAG-tagged wild-type PTPN18 and D197A were expressed in HepG2 cells. The HER2/PTPN18 complex formation was detected by co-immunoprecipitation at the indicated time points after EGF stimulation. (B) Co-immunoprecipitated HER2 in A was quantified and compared with the un-stimulated state. (C) Schematic representation of different PTPN18 truncations. (D) Interactions of HER2 with different truncations of wild-type PTPN18 or PTPN18 inactive mutant C229S. Different PTPN18 truncations were equally expressed in HepG2 cells. The PTPN18-associated HER2 was immunoprecipitated and detected by western blotting. (E) Direct interactions of HER2 with different PTPN18 truncations were examined by GST pull-down assay. Different GST-PTPN18C229S truncations were purified to homogeneity and applied to EGF-induced HepG2 cell lysates. After extensive washing, PTPN18-associated HER2 was analyzed by western blotting. (F) Bar graphs of the $K_{\text {cal }} / K_{\mathrm{m}}$ values of PTPN18 for the phospho-peptides derived from HER2 and EGFR (Related to Supplementary information, Table S1). All statistics shown represent the mean \pm SEM from at least three independent experiments. ${ }^{* *} P<0.01$ compared with control.

ficity. The calculated $K_{\text {cat }} / K_{\mathrm{m}}$ values of PTPN18 toward the three phospho-peptides were from $4 \times 10^{5}$ to $8 \times 10^{5}$ $\mathrm{M}^{-1} \mathrm{~s}^{-1}$, well within the range of physiological substrates tested for other known tyrosine phosphatases [30, 32, 33]. Biochemically, PTPN18 was a relatively poor phosphatase for small artificial substrates, such as para-nitrophenyl phosphate (pNPP), with a $K_{\text {cat }} / K_{\mathrm{m}}$ of $2.7 \mathrm{M}^{-1} \mathrm{~s}^{-1}$, 2000-fold lower than PTP1B, a prototype tyrosine phosphatase (Supplementary information, Figure S2B and S2C). The catalytic activity of PTPN18 towards the three phosphorylated peptides of HER2 was five orders of magnitude higher than towards the single phenyl-ring substrate, suggesting that the residues flanking the HER2 phosphorylation sites are among the determinants of the 
recognition of these sites by PTPN18.

Overall structures of PTPN18 in complex with specific HER 2 tyrosine-phosphorylated peptides

The above cellular and biochemical experiments unequivocally revealed that PTPN18 specifically dephosphorylates HER2 at the $\mathrm{pY}^{1112}, \mathrm{pY}^{1196}$ and $\mathrm{pY} \mathrm{Y}^{1248}$ sites. To define the structural basis of the PTPN18-specific recognition of distinct HER2 phosphorylation sites, we solved the crystal structures of the catalytically inactive mutant PTPN18-C229S in complex with HER2-pY $Y^{1248}$ at $2.0 \AA$, $\mathrm{pY}^{1196}$ at $2.2 \AA$ and $p \mathrm{Y}^{1112}$ at $2.5 \AA$ (Supplementary information, Table S2). All three HER2 phosphorylated peptides bound to PTPN18 in an extended conformation at the $\mathrm{N}$-terminus, perpendicular to the five central $\beta$-sheets, as did the previously solved structure of PTP1B C215A in complex with insulin receptor activation segments; however, these peptides assumed different conformations in the C-terminus (Figure 3A and Supplementary information, Figure S3). The HER2-pY ${ }^{1112}$ peptide has an "S" shape, whereas HER2-pY $Y^{1196}$ and HER2-pY ${ }^{1248}$ exhibited a $\beta$-turn configuration (Figure 3A). Binding of the peptide also triggered a conformational change in PTPN18. In particular, binding of the HER2 phospho-peptide induced the closure of the WPD loop in which the tip traveled $6.2 \AA$ to precisely position the catalytically invariant Asp for efficient catalysis as observed in PTP1B, YopH and several other PTPs. However, no such changes have been observed in LYP, another PTP member of the PEST family, in complex with an inhibitor or peptides (Figure 2A) $[28,30]$.

Detailed structure of PTPN18 in complex with HER2 phospho-peptides (ENPEp $Y^{1248}$ LGLD and ENPEpY $Y^{1196}$ $L T P Q)$

In the structure of PTPN18-C229S in complex with the HER2 $\mathrm{pY}^{1248}$ peptide (Ac-ENPEpY ${ }^{1248}$ LGLD-NH $_{2}$ ), seven residues (1246-1252) were unambiguously assigned to the Fo-Fc omitting electron-density map (Figure 3B). The D64, K63 and a water molecule coordinated by R138 from PTPN18 composed five backbone H-bonds of the HER $2 \mathrm{pY}^{1248}$ peptide and positioned the peptide along specific PTPN18 surface loops, which were the phosphotyrosine recognition loop, the P-loop, the WPD loop, the Q loop and the $\alpha-1$ helix from $\mathrm{N}$ - to the C-terminus (Figure 3A and 3C and Supplementary information, Figure $\mathrm{S} 4 \mathrm{C}$ ).

In the core region of contact between the HER2 $\mathrm{pY}^{1248}$ peptide and PTPN18, the central $\mathrm{pY}$ inserts into the active site, forming eight hydrogen bonds with PTPN18. The phenyl ring of the HER2 $\mathrm{pY}^{1248}$ is packed by Y62, V65, R140 and Q276 of PTPN18. Outside of the
pY-binding pocket, the D64 accepts two hydrogen bonds from the two main-chain amides of the central $\mathrm{pY}^{1248}$ and L1249, defining the initial configuration of the peptide C-terminus (Figure 3C and Supplementary information, Figure S4C). Consistent with these observations, the mutation D64A did not change the catalytic activity of PTPN18 towards the small artificial substrate pNPP but significantly decreased the activity towards the HER2 $\mathrm{pY}^{1248}$ peptide (Figure 6B). In the N-terminus of the peptide, a hydrogen bond between the P1246 and the K63 of PTPN18 defines the peptide orientation, which positions the P1246 in the hydrophobic area formed by residue Y62 and the aliphatic carbons of K63. C-terminally to the central $\mathrm{pY}$, four residues of the HER2 $\mathrm{pY}^{1248}$ peptide form a $\beta$-turn, in which an internal hydrogen bond is formed between the L1249 backbone carbonyl and the D1252 backbone amide. The pY+1 L1249 of the HER2 $\mathrm{pY}^{1248}$ peptide sits in a well-defined hydrophobic pocket (Figure 4A). After the linker, G1250, L1251 and D1252 form extensive interactions with R198 from PTPN18 (Figure 3C and Supplementary information, Figure S4C). Relative to the HER2 $\mathrm{pY}^{1248}$ site, the HER2 $\mathrm{pY}^{1196}$ peptide (Ac-ENPE $\mathrm{pY}^{1196}$ LTPQ- $\mathrm{NH}_{2}$ ) shares five identical residues from the $\mathrm{N}$-terminal (pY-4)/E1192 to the C-terminal (pY+1/L1197) position. The $K_{\text {cat }} / K_{\mathrm{m}}$ of PTPN18 towards the HER2 $\mathrm{pY}^{1196}$ site is approximately half of its $K_{\text {cat }} / K_{\mathrm{m}}$ towards HER2 $\mathrm{pY}^{1248}$, which may explain the less specific interactions observed in the structure of the PTPN18-C229S in complex with the HER2 $\mathrm{pY}^{1196}$ peptide (Figure 3D and Supplementary information, Figure S4D). As expected, three residues of P1194, E1195 and central $\mathrm{pY}^{1196}$ complex of the PTPN18-pY ${ }^{1196}$ peptide assume positions identical to those in the PTPN18-pY $\mathrm{Y}^{1248}$ complex, and the pY+1 L1197 falls into the same hydrophobic pocket as L1249 (Figure 4B). Unlike the linker G1250 in the HER2 $\mathrm{pY}^{1248}$ peptide, the pY+2 T1198 of the HER2 $\mathrm{pY}^{1196}$ peptide forms an internal hydrogen bond with E1195, which distorts the peptide slightly outwards. After T1198, the pY+3 P1199 both engages in hydrophobic interactions and forms a hydrogen bond with R198 of PTPN18 (Figure 3D and Supplementary information, Figure S4D).

The structure of PTPN18 in complex with the HER2 phopho-peptide (PLQRpY $Y^{1112}$ SEDP)

Unlike the HER2 $\mathrm{pY}^{1248}$ and HER2 $\mathrm{pY}^{1196}$ peptides that form a $\beta$-turn at their $\mathrm{C}$-termini, the nine residues of the HER $2 \mathrm{pY}^{1112}$ peptide display an extended $\beta$-structure defined by seven hydrogen bonds along the peptide backbone, which are composed by residues of K63, D64, R198, C233, Q262 and R268 of PTPN18 (Figure 3A and $3 \mathrm{E}$ and Supplementary information, Figure S4E). The 
A

B

D
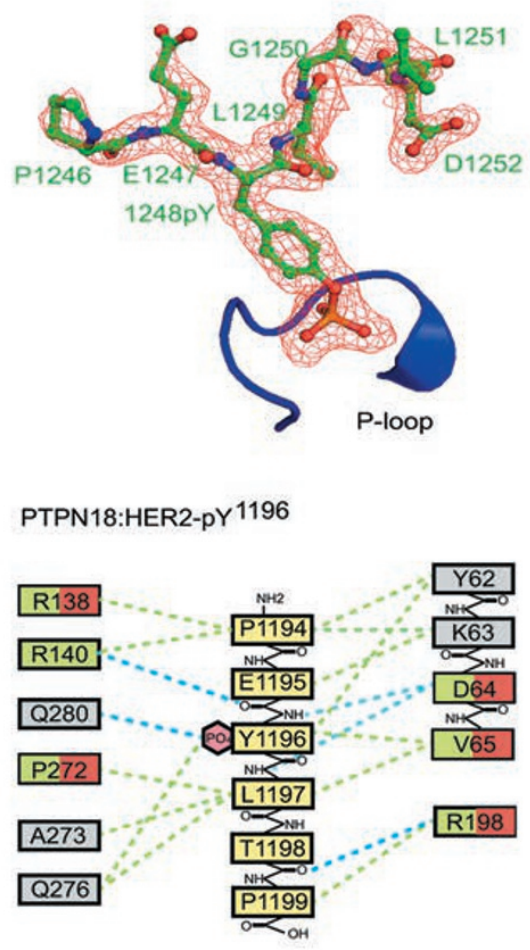

C

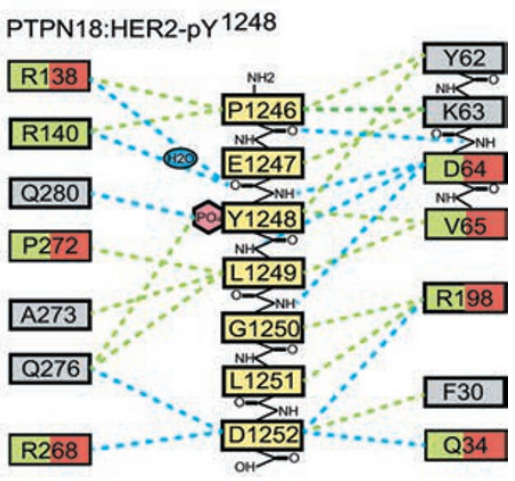

$\mathrm{E}$

\section{PTPN18:HER2-pY 1112}

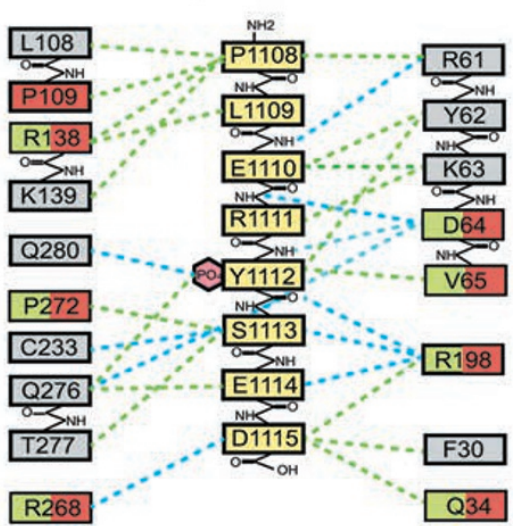

\footnotetext{
- Hydrophobic contacts

H-bonds

Mutation decreases PTPN18:HER2-pY $Y^{1248}$ or 1196 activity

Mutation decreases PTPN18:HER2-pY1112 activity
}

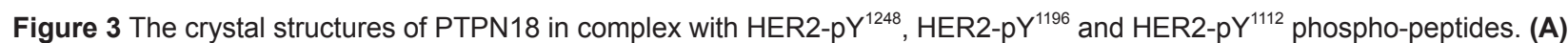

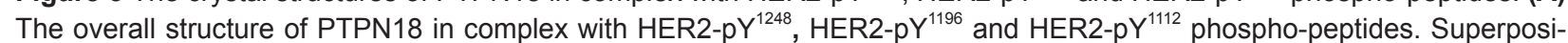
tion of the PTPN18-HER2-phospho-peptide structures on the PTPN18 native protein structure (PDB: 2OC3); the movement

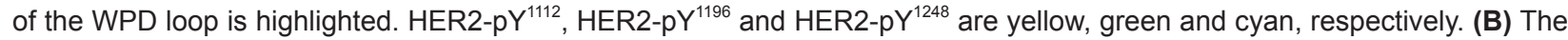
Fo-Fc annealing omit map (contoured at 3.0 $\sigma$ ) around HER2-pY $Y^{1248}$ phospho-peptides. (C-E) Schematic representation of in-

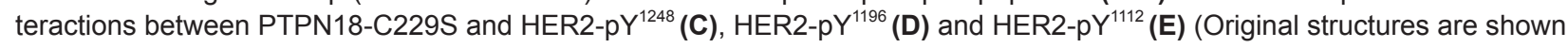
in Supplementary information, Figure S4C-S4E). The mutations that decreased the PTPN18 phosphatase activity toward only HER2-pY $Y^{1196}$ and HER2-pY $Y^{1248}$ are indicated in green, the mutations that decreased the PTPN18 activity toward only HER2$\mathrm{pY}^{1112}$ are indicated in red, and the mutations that decreased the PTPN18 activity toward all three phospho-peptides are indicated in half green and half red. The blue dashed lines represent hydrogen bonds. The green dashed lines dashed lines represent hydrophobic interactions. 
A

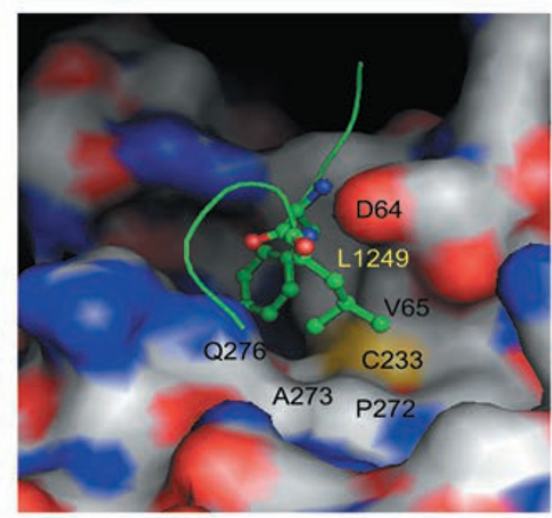

C

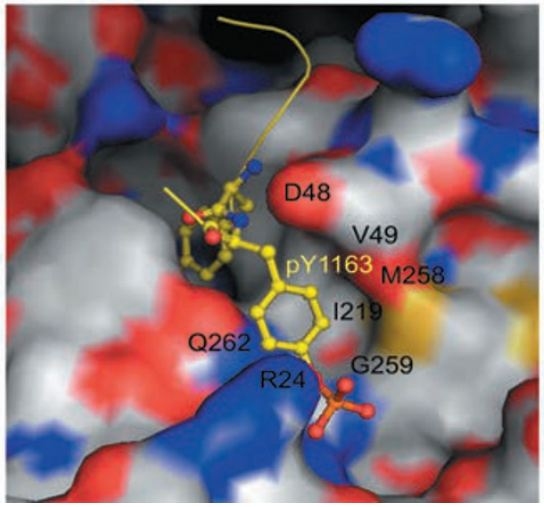

B

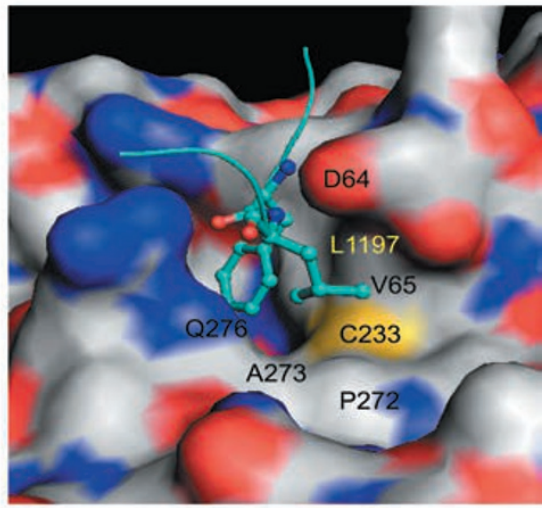

D

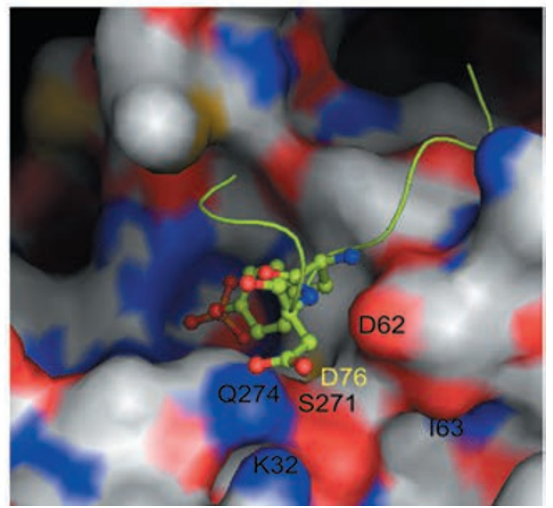

Figure 4 The hydrophobic PTPN18 pY+1 site is responsible for its substrate specificity. (A) The surface representation of the structure of the PTPN18-C229S- HER2-pY ${ }^{1248}$ peptide complex. The hydrophobic pocket encompasses V65, P272, A273 and Q276, with the residue C233 serving as the bottom. (B) The surface representation of the structure of the PTPN18-C229SHER2-pY ${ }^{1196}$ peptide complex. (C) The surface representation of the structure of the PTP1B-C215S-IRK-pY ${ }^{1162 / 1163}$ peptide complex (PDB: 1G1F). The "second pY-binding site" is composed by V49, G259, M258, Q262, R24, with the I219 as the bottom. The presence of G259 allowed the penetrance of the bulky phospho-tyrosine. (D) The surface representation of the structure of the LYP/C227S- SKAP-HOM pY ${ }^{75}$ peptide complex. (PDB: 3OMH). The LYP has a narrower pocket entrance.

internal electrostatic interactions between the side-chains of the pY-1 R1111 and pY+3 E1115 and several backbone hydrogen bonds also help to stabilize the extended $\beta$ conformation of the HER2 $\mathrm{pY}^{1112}$ peptide (Supplementary information, Figure S4E).

$\mathrm{N}$-terminally to the central binding core of $\mathrm{pY}$, the pY-1 R1111 and pY-2 E1110 have non-specific hydrophobic interactions with K63 and D64. The pY-3 L1109 sits in a pocket defined by Y62 and R138 from PTPN18 and $\mathrm{pY}^{1112}$ from HER2 (Figure 3E and Supplementary information, Figure S4E). The N-terminal pY-4 P1108 reaches a hydrophobic region defined by L108, P109 and the aliphatic parts of R61 and K139 from PTPN18 (Figure $3 \mathrm{E}$ and Supplementary information, Figure S4E). The hydrophobic interactions of PTPN18 with P1108 and L1109 of HER2 are unique in defining PTPN18 and HER2 $\mathrm{pY}^{1112}$ phospho-peptide interactions, as HER2 $\mathrm{pY}^{1196}$ and HER2 $\mathrm{pY}^{1248}$ have the polar residues Glu1192/1244 and Asn1193/1245 in the corresponding positions. Consistently, the mutation P109A, which reduces the hydrophobic interactions of these residues, decreased the ability of PTPN18 to dephosphorylate the HER2 $\mathrm{pY}^{1112}$ peptide, but not the HER2 $\mathrm{pY}^{1196}$ or $\mathrm{pY}^{1248}$ peptides. The R138L mutation, which maintained its hydrophobic property, decreased HER2 $\mathrm{pY}^{1196}$ and $\mathrm{pY}^{1248}$ peptide hydrolysis but had little effect on HER2 $\mathrm{pY}^{1112}$ peptide hydrolysis (Figures $3 \mathrm{E}$ and $6 \mathrm{~A}-6 \mathrm{~B})$.

In the $\mathrm{C}$-terminus, the $\mathrm{pY}+1$ position of HER2 $\mathrm{pY}^{1112}$ peptide has an $\mathrm{S} 1113$ instead of a hydrophobic residue. A hydrogen bond between the carbonyl group of the 
A

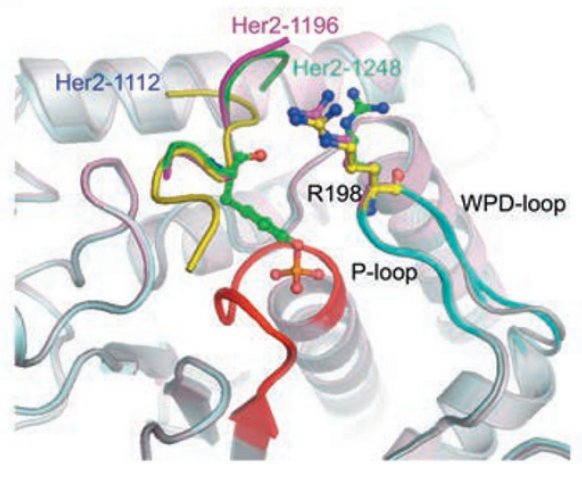

C

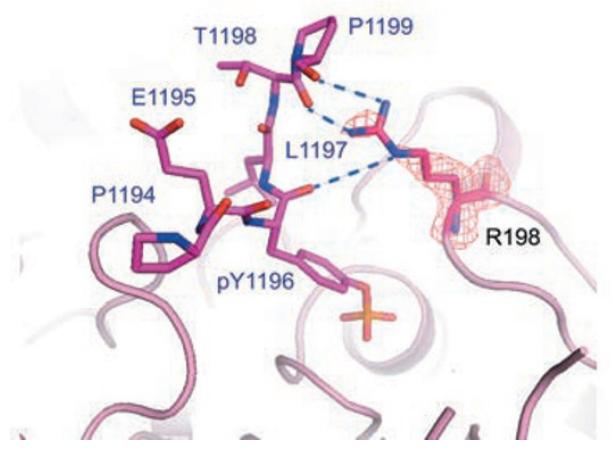

B

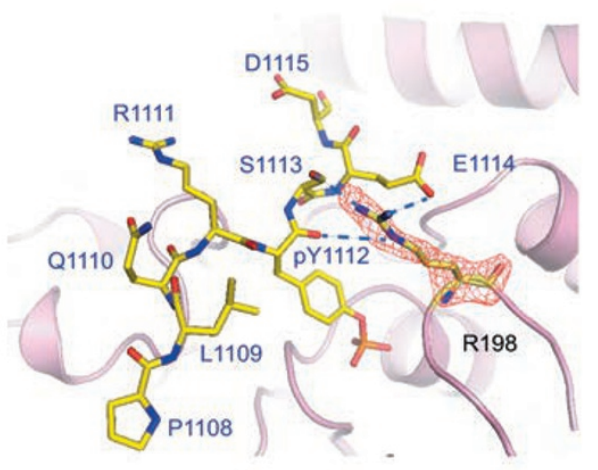

D

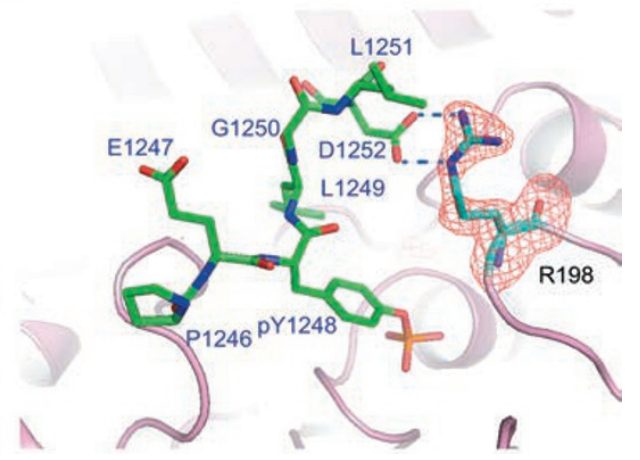

Figure 5 The side-chain plasticity of the R198 residue allows PTPN18 to recognize different peptide conformations. (A) Superposition of three PTPN18-HER2 peptide complex structures. The side-chain plasticity of R198 is highlighted. (B) The extended conformation of R198 in the structure of the PTPN18-HER2-pY ${ }^{1112}$ complex. (C) R198 conformation in the complex structure of PTPN18-HER2-pY ${ }^{1196}$. (D) R198 assumed a different conformation in the complex structure of PTPN18-HER2$\mathrm{pY}^{1248}$.

backbone S1113 and the sulphur of $\mathrm{C} 233$ defines the backbone conformation, and the side-chain of the S1113 engages in a hydrogen bond with the guanine group of R198, which assumes a stretched conformation (Figure $3 \mathrm{E}$ and Supplementary information, Figure S4E). The main-chain amide of pY+2 E1114 is recognized by Q276 and its side-chain forms hydrogen bond with R198. The last C-terminal residue P1116 forms extensive hydrophobic interactions with Q34 and F30 from helix $\alpha 1$, and its main-chain carbonyl forms strong hydrogen bonds with R268 (Figure 3E and Supplementary information, Figure S4E). Consistently, the elimination of pY+4 P1116 from the peptide abolished PTPN18 activity towards the peptide (Supplementary information, Table S1). In addition, the mutations Q34A or R268A significantly reduced the activity of PTPN18 towards the HER2 $\mathrm{pY}^{1112}$ phospho-peptide, revealing an essential role for the region close to the "LYP-specific insert" in binding PTPN18 substrates (Figures 3E, 6A, 6B and Supplementary information, Figure S4E).
The hydrophobic PTPN18 $p Y+1$ site

The structures of both the HER2 $\mathrm{pY}^{1248}$ and HER2 pY ${ }^{1196}$ peptide-PTPN18-C229S complexes revealed a hydrophobic site for packing the $\mathrm{pY}+1$ Leu residue. The hydrophobic site is surrounded by the hydrophobic residue V65 from the "pY-binding loop", P272, A273 and the aliphatic carbon chain of Q276 from the "Q-loop", with the "P-loop" residue C233 serving as the bottom (Figure 4A, 4B and Supplementary information, Figure $\mathrm{S} 5 \mathrm{~A}$ and $\mathrm{S} 5 \mathrm{~B}$ ). This hydrophobic site is reminiscent of the "second pY-binding site" of PTP1B in the complex structures of the PTP1B-C215A-insulin receptor segment and PTP1B-C215S-BPPM (Figure 4C and Supplementary information, Figure S5C) [32, 34, 35]. Unlike PTP1B, which favors a second $\mathrm{pY}$ C-terminally to the central $\mathrm{pY}$, PTPN18 does not prefer $\mathrm{pY}$ at the $\mathrm{pY}+1$ position, as illustrated by the very weak activity of PTPN18 towards the HER2 $\mathrm{pY}^{1221} \mathrm{pY}^{1222}$ site (Figures 1B, 1C, 1E and 2F). Relative to the second PTP1B pY-binding site, two key substitutions in PTPN18 explain the observed difference 
A

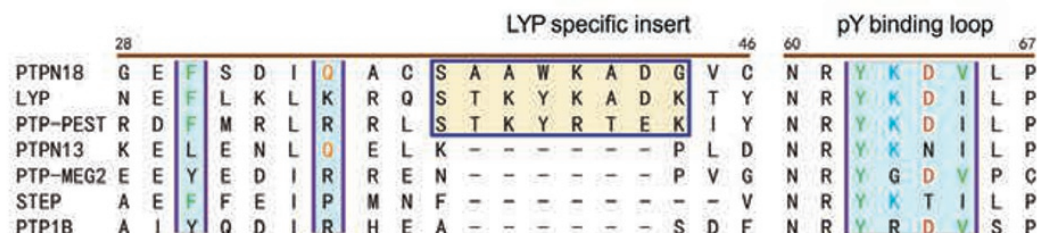

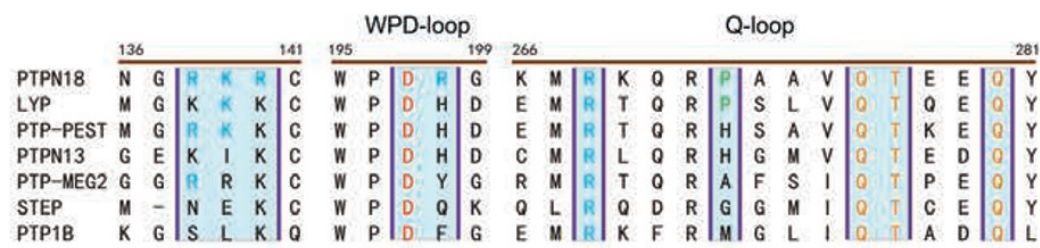

B

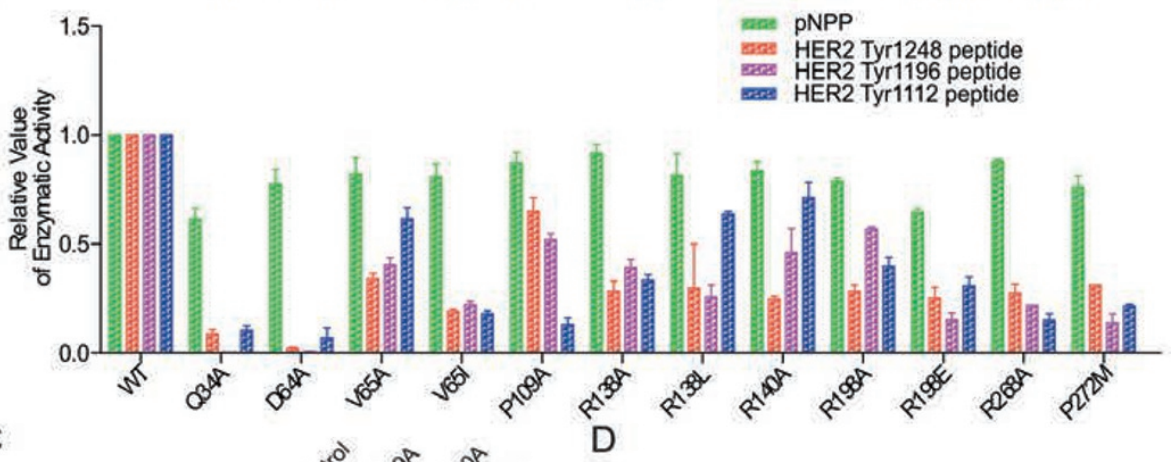

EGF

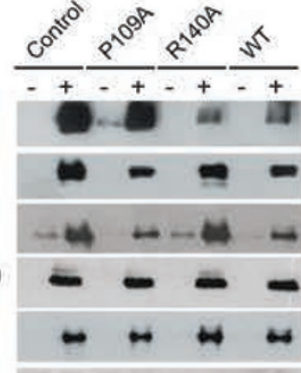

IB:Total-HER2

IB:p-AKT(308)

IB:p-AKT(473)

IB:Total-AKT

IB:p-ERK(202/204)

IB:Total-ERK

IB:p-SRC(416)

IB:p-SRC(527)

IB:Total-SRC

IB:p-Paxillin(Y118)

IB:Total-Paxillin

IB:pPAK1(S144)

IB:pPAK1(T423)

IB:Total-PAK1

IB:FLAG

IB: $\beta$-ACTIN
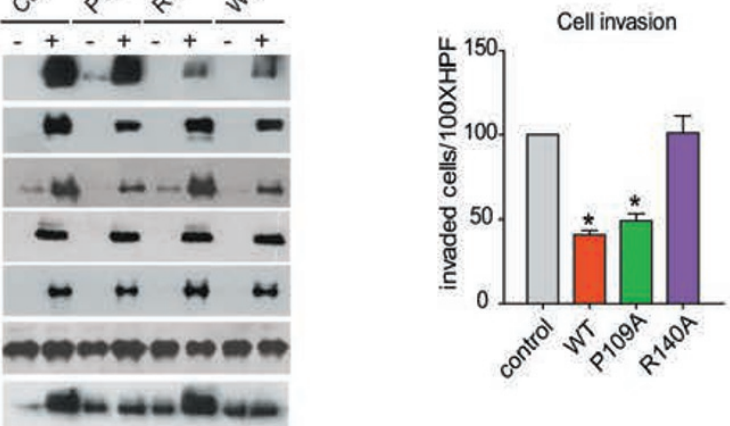

E
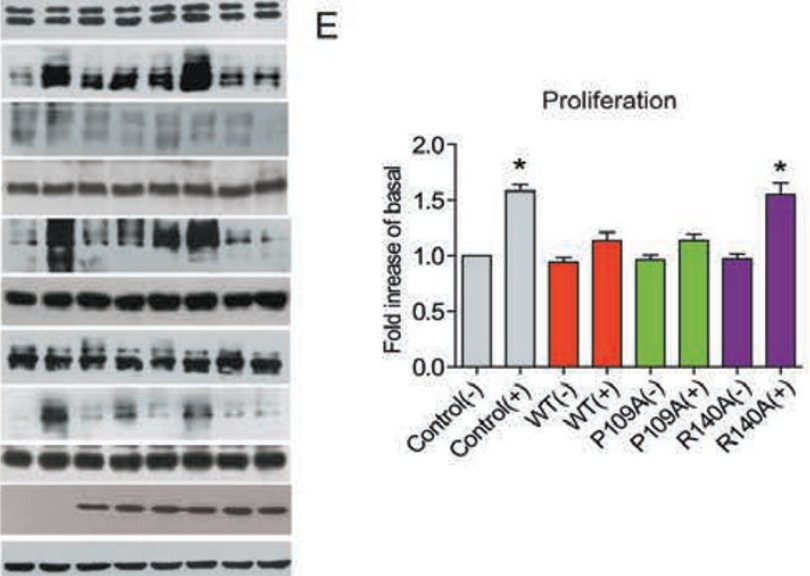
in substrate selectivity. The replacement of R24 in PTP1B with Q34 in PTPN18 decreases the basic potential for targeting the second $\mathrm{pY}$, and the change of G259 in PTP1B to A273 in PTPN18 blocks the channel linking the phosphate to the phenyl ring (Figures $4 \mathrm{~A}-4 \mathrm{C}$ and $6 \mathrm{~A}$, Supplementary information, Figure S2A-S2C). Whereas the electron density of $\mathrm{pY}^{1163}$ in the phenyl ring of $\mathrm{pY}+1$ in the PTP1B-IRK segment complex is disordered, the $\mathrm{pY}+1$ Leu of the HER2 $\mathrm{pY}^{1248}$ or HER2 $\mathrm{pY}^{1196}$ is buried deeper in the apolar pocket and has a defined electron density [32]. This effect is mostly due to the shorter sidechain of C233 in PTPN18 compared with I219 in PTP1B at the bottom of the hydrophobic pocket (Figure 4A-4C and Supplementary information, Figure S4A-S4D).

Unlike PTPN18, another PEST-PTP family member, LYP, prefers acidic residues at the $\mathrm{pY}+1$ position [30]. LYP has a narrower pocket entrance, defined by the relatively longer side-chains of I63 and S271, compared with V65 and A273 in PTPN18 (Figures 4A, 4D, 6A and Supplementary information, Figure S5D). As a result, the pY+1 Asp of SKAP-HOM floats above the LYP-binding pocket.

Consistent with the above observations, the mutation of the PTPN18 residue V65 to alanine (V65A), V65 to the corresponding LYP residue I63 (V65I) and P272 to the corresponding PTP1B residue M258 (P272M) decreased PTPN18 activity towards the HER2 $\mathrm{pY}^{1248}$ and $\mathrm{pY}^{1196}$ peptides by $>2$ - fold (Figure 6A-6B). The residues surrounding this hydrophobic pocket, V65, C233, P272 and A273, are not conserved in most PTP family members (Figure 6A and Supplementary information, Figure S6). Therefore, the crystal structure and mutagenesis studies revealed that PTPN18 has a special pocket favoring a hydrophobic $\mathrm{pY}+1$ residue and that this feature is a determinant of its substrate selectivity.

The side-chain plasticity of PTPN18 R198 enables two different peptide conformations
In the PTPN18-HER2 $\mathrm{pY}^{1112}$ complex structure, the R198 of PTPN18 assumes an extended conformation and the guanine group is engaged in favorable hydrogen bonds with the side-chains of $\mathrm{pY}+1 \mathrm{~S} 1113$ and $\mathrm{pY}+2$ E1114 (Figure 5A and 5B). In the complex structure of PTPN18 and HER2 pY ${ }^{1196}$, R198 assumes a similar extended conformation that forms two hydrogen bonds with peptide main-chain carbonyl groups and hydrophobic interactions with pY+3 P1199 (Figure 5A and 5C). When PTPN18 is in complex with the HER2 $\mathrm{pY}^{1248}$ peptide, the presence of $\mathrm{L} 1251$ at the $\mathrm{pY}+3$ position prohibits the extended R198 conformation and the $\chi 3$ of R198 switches from $180^{\circ}$ to $48^{\circ}$, enabling the guanine group to form a defined salt bridge with the side-chain of pY+4 D1252 from the HER2 $\mathrm{pY}^{1248}$ peptide (Figure 5A and 5D).

Therefore, the side-chain plasticity of R198 enables PTPN18 to accommodate two types of peptide sequences and secondary structures in the C-terminus, a $\beta$-turn with $p Y+3$ Leu and $p Y+4$ Asp and an "S" shape with $p Y+1$ Ser and $\mathrm{pY}+2 \mathrm{Glu} / \mathrm{Asp}$ (Figure 5A). The hydrogen bonds and charge-charge interactions between the peptides and the guanine group of R198 determine PTPN18 and phospho-peptide interactions. Consistent with these observations, eliminating the side-chain of R198 to Ala or changing it to negatively charged Glu decreased PTPN18 activity towards the HER2 $\mathrm{pY}^{1248}$ or $\mathrm{pY}^{1112}$ peptides by 2-3-fold (Figure 6A-6B).

Specific interactions between PTPN18 and HER2 $p Y^{1196}$ and $p Y^{1248}$ contribute to the regulation of the HER2-mediated phosphorylation cascade

HER2 is a potent upstream regulator of EGF-induced cell proliferation and migration. Two of the PTPN18-regulated HER2 phosphorylation sites, $\mathrm{pY}^{1196}$ and $\mathrm{p} \mathrm{Y}^{1248}$, mediate cell growth and migration via the activation of the phosphorylation cascades, including, but not limited to DOKR-ERK, PI3K-AKT-WT1, SRC-Paxillin-PAK and other cascades $[15-17,20,36]$. Therefore, PTPN18 is

Figure 6 Kinetic analysis of PTPN18-HER2 phospho-peptide interfaces and the regulation of the cellular function and signal transduction cascades by specific interactions between PTPN18 and the HER2 $\mathrm{pY}^{1196}$ and $\mathrm{pY}^{1248}$ sites. (A) Sequence alignment of PTPN18 with PEST-family PTP and other PTPs reported to be involved in HER2 dephosphorylation. Residues involved in PTPN18 and HER2 interactions are highlighted and compared with close PTP members. (B) The catalytic activity of wild-type PTPN18 and various mutants towards pNPP and the HER2 phospho-peptides $\mathrm{pY}^{1112}, \mathrm{pY}^{1196}$ and $\mathrm{pY} \mathrm{Y}^{1248}$. $\mathrm{pNPP}$, green; $p Y^{1112}$, blue; $p Y^{1196}$, magenta; $p Y^{1248}$, red. (C) Effects of PTPN18-WT, PTPN18-HER2-pY $Y^{1196}$ and -pY ${ }^{1248}$-biased mutant R140A and the PTPN18-HER2-pY ${ }^{1112}$-biased mutant P109A on the EGF-induced phosphorylation cascade involved in cell proliferation and invasion. Equal amounts of PTPN18-WT, P109A and R140A were overexpressed in HepG2 cells, and their effects on EGF-induced phosphorylation of HER2 $Y^{1112}, Y^{1139}, Y^{1196}, Y^{1221 / 1222}$ and $Y^{1248}$; AKT $S^{308}$ and AKT $S^{473}$, ERK $T^{202} Y^{204}$; SRC $Y^{416}$ and SRC $Y^{527}$; paxillinp $Y^{118}$ and PAK1 $S^{144}$ and $T^{423}$ were examined. $p$-HER2, p-AKT and p-ERK were monitored 5 min after EGF stimulation, and levels of p-SRC, p-paxillin and p-PAK1 were monitored 30 min after EGF stimulation. (D) The effects of the PTPN18 biased mutants P109A or R140A on EGF-induced cell invasion. All statistics shown represent the mean \pm SEM from at least three independent experiments. ${ }^{*} P<0.05$ compared with control. (E) The effects of the PTPN18 P109A or R140A mutants on EGF-induced cell proliferation. ${ }^{*} P<0.05$ compared with non-EGF-stimulated cells. 
likely to regulate cell growth and invasion through its action on HER2 phosphorylation. Knockdown of PTPN18 and PTP-MEG2, two known HER2 phosphatases, promoted EGF-mediated cell growth and invasion. Conversely, overexpression of PTPN18 inhibited EGF-induced cell proliferation and invasion (Supplementary information, Figure S7A-S7B).

Next, we evaluated the importance of the specific interactions between PTPN18 and HER2 $\mathrm{Y}^{1196}$ and $\mathrm{Y}^{1248}$ phosphorylated sites in EGF-induced HER2 signaling, utilizing information derived from complex crystal structures. In PTPN18, P109 of PTPN18 forms strong hydrophobic interactions with P1108 of the HER2 $\mathrm{pY}^{1112}$ phospho-peptide, but has no direct role in binding to HER2 $\mathrm{pY}^{1196}$ and $\mathrm{pY}^{1248}$ phospho-peptides. The mutation of P109A significantly reduced the activity of PTPN18 towards the HER2 $\mathrm{pY}^{1112}$ phospho-peptide, but had little effect on its activity towards the HER2 $\mathrm{pY}^{1196}$ or $\mathrm{pY}^{1248}$ phospho-peptides (Figure 6B). Conversely, the mutation of the specific $\mathrm{pY}^{1248}$-interacting residue $\mathrm{R} 140$ of PTPN18 to Ala (R140A) displayed strong activity towards the HER2 $\mathrm{pY}^{1112}$ but decreased activity towards HER2 $\mathrm{pY}^{1196}$ and $\mathrm{pY}^{1248}$ (Figure 6B). Therefore, R140A was a biased PTPN18 mutant that only regulated the HER2 $Y^{1196}$ and $Y^{1248}$ phosphorylation states, whereas P109A was an HER2 $\mathrm{pY}^{1112}$ biased mutant.

We evaluated these biased mutants by monitoring their effects on EGF-induced changes in HER2 phosphorylation. Consistent with kinetic studies, P109A only decreased the HER2 $\mathrm{Y}^{1196}$ and $\mathrm{Y}^{1248}$ phosphorylation, whereas R140A specifically downregulated the HER2 $\mathrm{Y}^{1112}$ phosphorylation state (Figure 6C). Neither mutant had significant effects on the HER2 $\mathrm{Y}^{1139}$ or $\mathrm{Y}^{1221 / 1222}$ phosphorylation. Next, we monitored the altered phosphorylation cascades downstream of the HER2 activation (Figure 6C). Overexpression of PTPN18-WT significantly decreased the EGF-induced phosphorylation of $\mathrm{ERK}^{202 / 204}, \mathrm{AKT}^{308}, \mathrm{AKT}^{473}, \mathrm{SRC}^{416}, \mathrm{SRC}^{527}$, Paxillin $^{118}$ and $\mathrm{PAK}^{423}$, but did not significantly change the phosphorylation of PAK $^{144}$. These phospho-proteins are not likely to be the direct substrates of PTPN18, as PTPN18 displays weak activity towards corresponding phospho-peptides (Supplementary information, Table S1). Overexpression of HER2-pY $\mathrm{Y}^{1112}$ biased mutant P109A of PTPN18 exerts similar effects to the wild type; however, overexpression of R140A, which perturbs HER2-pY ${ }^{1196}$ and $\mathrm{pY}^{1248}$, had much weaker effects compared with the wild type or P109A mutant (Figure 6C). Consistent with the change in the phosphorylation cascade, overexpression of wild-type or the P109A mutant suppressed the EGF-induced cell proliferation and invasion, whereas the R140A mutant had no such effect (Figure 6D and 6E).
Taken together, these biochemical and cellular studies confirm that unique structural features of PTPN18 are responsible for recognition of the HER2 $\mathrm{Y}^{1196}$ and $\mathrm{Y}^{1248}$ phosphorylation sites, which determine HER2-mediated cell growth and invasion by regulating the phosphorylation cascade downstream of HER2.

\section{PTPN18 promoted K48-linked polyubiquitination and proteasomal degradation of HER2}

Unlike the HER2-Y ${ }^{1196}$ and - $\mathrm{Y}^{1248}$ phosphorylation that mediate cell invasion and proliferation, the phosphorylation of HER2-Y $\mathrm{Y}^{112}$ by antibody treatment recruited the c-Cbl ubiquitin ligase and shuttled the HER2 protein to the lysosome for degradation [21]. Therefore, knockdown of PTPN18 should promote HER2 degradation by increasing its interaction with c-Cbl. Unexpectedly, EGF-induced HER2 degradation was significantly decreased, rather than increased, by knockdown of endogenous PTPN18 (Figure 7A). In comparison, the knockdown of another HER2 phosphatase, PTP-MEG2, had little effect on the HER2 protein level. Conversely, overexpression of PTPN18, but not PTP-MEG2, augmented EGF-induced HER2 degradation (Figure 7B). These results demonstrate that PTPN18 is a negative regulator of HER2 stability. We then examined whether PTPN18 regulated HER2 degradation through ubiquitination. Knockdown of PTPN18 decreased EGF-induced HER2 ubiquitination, whereas overexpression of PTPN18 increased HER2 ubiquitination(Figure 7C-7D and Supplementary information, Figure S8A-S8B). Therefore, PTPN18 promoted HER2 degradation by upregulating HER2 ubiquitination.

Next, we examined whether proteasome or lysosome pathway underlies PTPN18-promoted HER2 degradation. Application of the proteasome inhibitor MG132 significantly increased the endogenous HER2 expression level and almost abolished the effects of PTPN18 overexpression (Figure 7E). Whereas EGF stimulation promoted the co-localization of HER2 to lysosome marker Lamp1, overexpression of PTPN18 did not significantly increase the co-localized portion. In contrast, more HER2 co-localized with RPN12, the proteasome marker, after the overexpression of PTPN18 (Figure 7F). These data suggest that PTPN18 promotes proteasome targeting and degradation of HER2.

The ubiquitination-linked lysine positions determined the specific topology of polyubiquitin chains and produced different cellular outcomes for the polyubiquitinated protein (Figure 7G). Whereas K63-linked polyubiquitin targets the Cbl-EGFR complex to the lysosome, K48- or K29-linked polyubiquitination might deliver the protein to the proteasome for degradation [37]. We then 
applied various lysine mutations of ubiquitin to determine which polyubiquitin chain mediates the effects of PTPN18 on HER2 ubiquitination (Figure 7G-7I). The co-expression of ubiquitin mutant K48R or UB-63, in which all lysines except K63 are mutated to R, resulted in a marked decrease in PTPN18-mediated HER2 ubiquitination. Conversely, overexpression of K63R mutant or UB-48 ubiquitin construct had little effect on HER2 ubiquitination (Figure 7H-7I). Taken together, these data suggest that PTPN18 promotes the proteasome-dependent degradation of HER2 through K48-linked polyubiquitination.

Phosphorylation of the "DQSPAGS" motif in the PTPN18 PEST domain recruited E3 ligase $\beta$-Trcp and mediated HER2 ubiquitination

To determine the region of PTPN18 that is required for specific HER2 ubiquitination, we used a series of PTPN18 truncations or mutant (Figure 8A). Overexpression of the CD and the 1-348, 1-405 and 1-420 truncations of PTPN18 failed to promote HER2 ubiquitination, whereas overexpression of the PEST domain of PTPN18 and PTPN18 truncations containing the $1-425$ region promoted HER2 ubiquitination (Figure $8 \mathrm{~B}$ and $8 \mathrm{C}$ ). Thus, a specific motif encompassing 415 to 425 was the key in PTPN18-mediated HER2 ubiquitination. In some PEST domain-containing proteins, such as VEGFR, the phosphorylation of a specific serine residue in the phosphodegron motif is essential for the recruitment of F-box E3-containing ligases and efficient ubiquitination [38]. A similar mechanism might also underlie PTPN18-mediated HER2 ubiquitination. To elucidate the role of the serine in the specific 415-425 motif of PTPN18, we mutated S419 and S423 to Ala and tested the effect of these mutantions on HER2 ubiquitination. Mutation of either S419A or S423A significantly decreased and double mutation of S419A/S423A completely abolished PTPN18-mediated HER2 ubiquitination (Figure 8D and $8 \mathrm{E})$.

To further test whether PTPN18 regulates HER2 ubiquitination through a phosphorylation-dependent mechanism, we examined the effects of several kinase inhibitors. Whereas the ERK or GSK3 inhibitor had little effect on HER2 ubiquitination, the AKT inhibitor significantly blocked HER2 ubiquitination (Figure 8F and 8G). Because "PADQSPAGSGAY" is not an AKT-favored peptide substrate sequence, whether AKT or another kinase downstream of AKT directly phosphorylates the S419 or S423 residues of PTPN18 requires further investigation. In addition, activation of AKT is downstream of activation of HER2. Therefore, a negative feedback loop of "activation of HER2-AKT-PTPN18-HER2 degradation" exists in HER2 regulation.

We also noticed that the "DQSPAGS" motif in PTPN18 was similar to the known $\beta$-Trcp recognition sequence of Cdc25B (DSGFCLDS), PDCD4 (DSGRGDS) and DLG (DSGLPS) [39]. Therefore, the phosphorylation of PTPN18 on its DQSPAGS motif may create a docking site for ubiquitin E3 ligases, $\beta$-Trcp. Consistent with this hypothesis, the full-length PTPN18 interacted with $\beta$-Trcp whereas the catalytic region of PTPN18 did not (Figure $8 \mathrm{H}$ ). Whereas mutation of S419A diminished $\beta$-Trcp binding, the S423A mutation or S419A/S423A double mutation almost abolished $\beta$-Trcp binding (Figure 8I). Moreover, knockdown of $\beta$-Trcp blocked PTPN18-mediated HER2 degradation (Figure 8J and 8K). Therefore, PTPN18 functions as an ancillary protein to recruit E3 ligase $\beta$-Trcp for HER2 ubiquitination.

PTPN18 regulates degradation kinetics of HER 2 and functions as a switch between lysosome routing and proteasome targeting

To define the role of the dephosphorylation of the HER2 $\mathrm{pY}^{1112}$ site in PTPN18-promoted HER2 degradation, we overexpressed PTPN18-WT and the D197A trapping mutant and compared the degradation kinetics of HER2. The PTPN18-WT and PTPN18 dominant-negative mutant D197A had similar effects on HER2 degradation at the early time point (4 h) (Figure 9A and Supplementary information, Figure S9A). After 4 h, PTPN18 D197A had stronger effect (from 8 to $12 \mathrm{~h}$; Figure 9A and Supplementary information, Figure S9A). We then checked the effect of PTPN18-D197A mutant on HER2 ubiqutination and $\mathrm{pY}^{1112}$ phosphorylation of ubiquitnated HER2. Overexpression of PTPN18-WT decreased the HER2 $\mathrm{Y}^{1112}$ phosphorylation in the ubiquitinated HER2 fractions, whereas overexpression of the PTPN18 D197A mutant significantly increased the phosphorylation of $\mathrm{Y}^{1112}$ (Figure 9B). Consequently, more HER2 were ubiquitinated with overexpression of the D197A mutant (Figure 9B and Supplementary information, Figure S9B). Next, we monitored the cellular distributions of HER2 with overexpression of PTPN18-WT or the D197A mutant. Whereas the distributions of HER2 to proteasomes were similar between the wild type and the D197A mutant, more HER2 proteins co-localized with Lamp1 in response to D197A overexpression (Figure 9C). Therefore, the phosphatase activity of PTPN18 controlled HER2 degradation kinetics through regulation of $\mathrm{Y}^{1112}$ phosphorylation and HER2 lysosome targeting.

Previous studies have demonstrated that $\mathrm{c}-\mathrm{Cbl}$ binding to the phosphorylated HER2 $\mathrm{Y}^{1112}$ site is required for lysosome routing [21]. After EGF stimulation, formation of the HER2/c-Cbl complex significantly increased in 
A
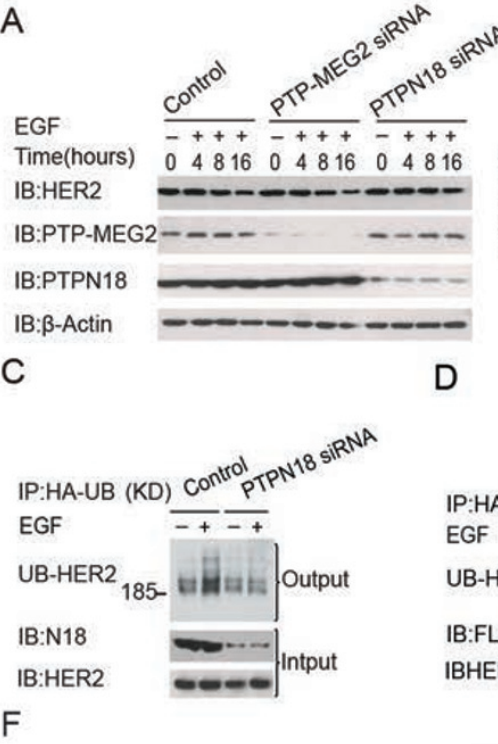

D
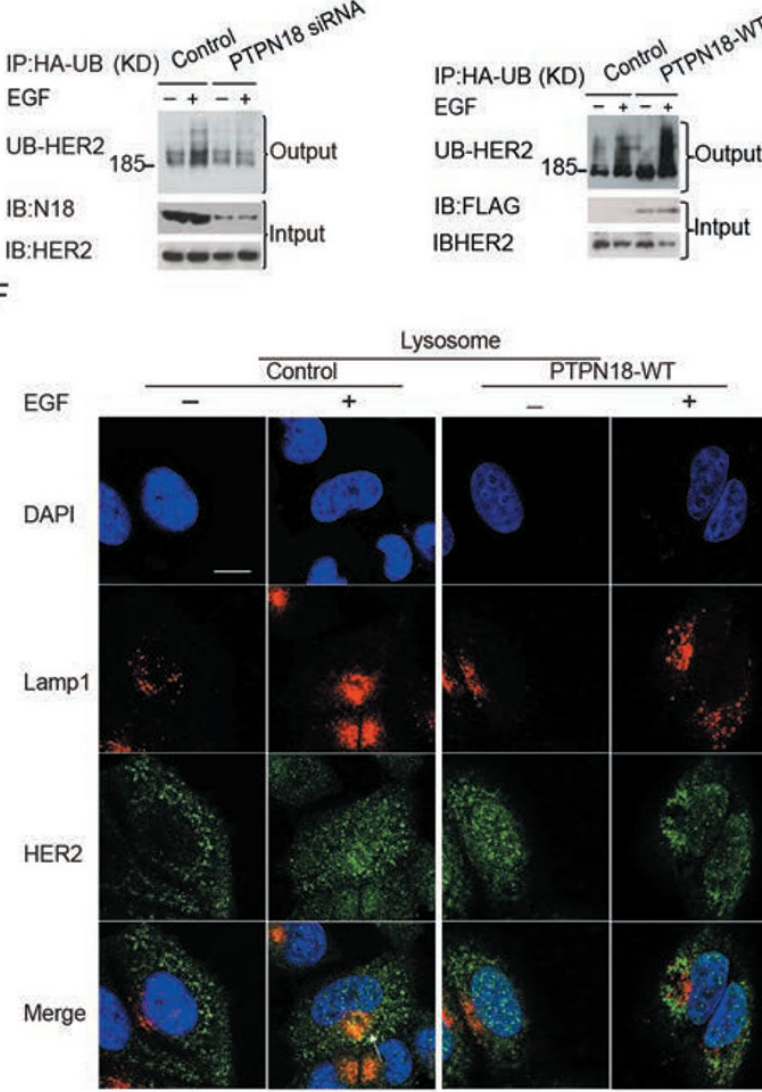

G

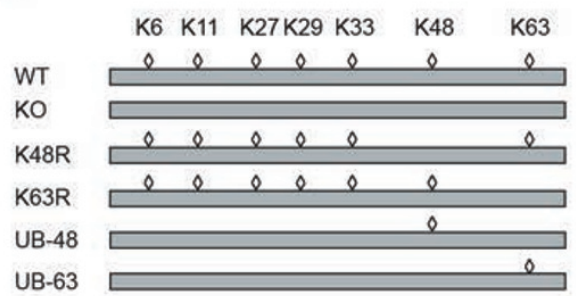

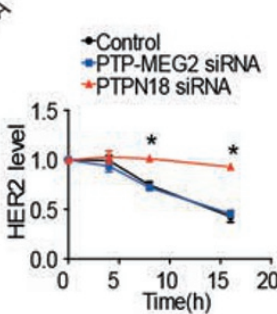

BHER2
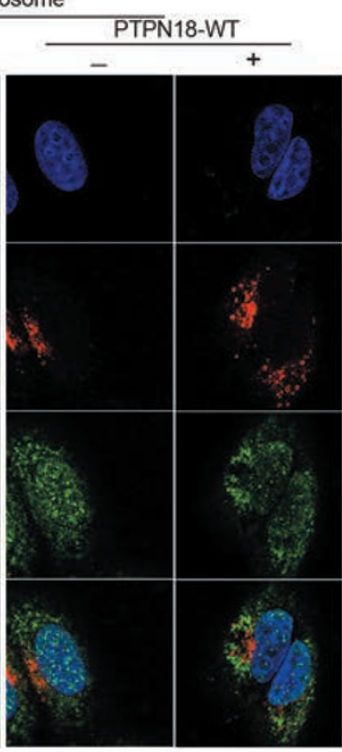

$\mathrm{H}$
B

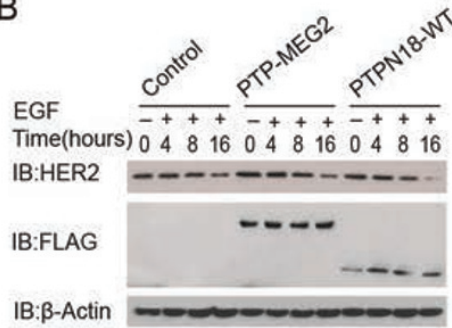

E

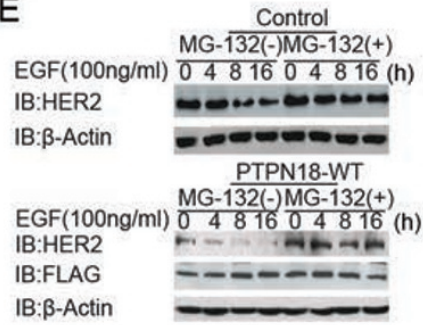

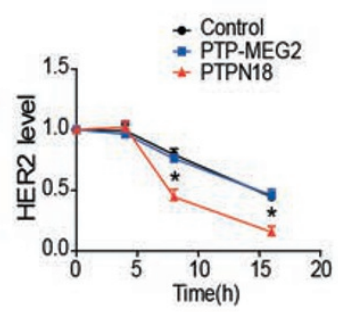

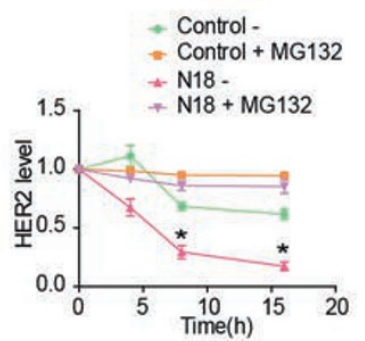

EGF
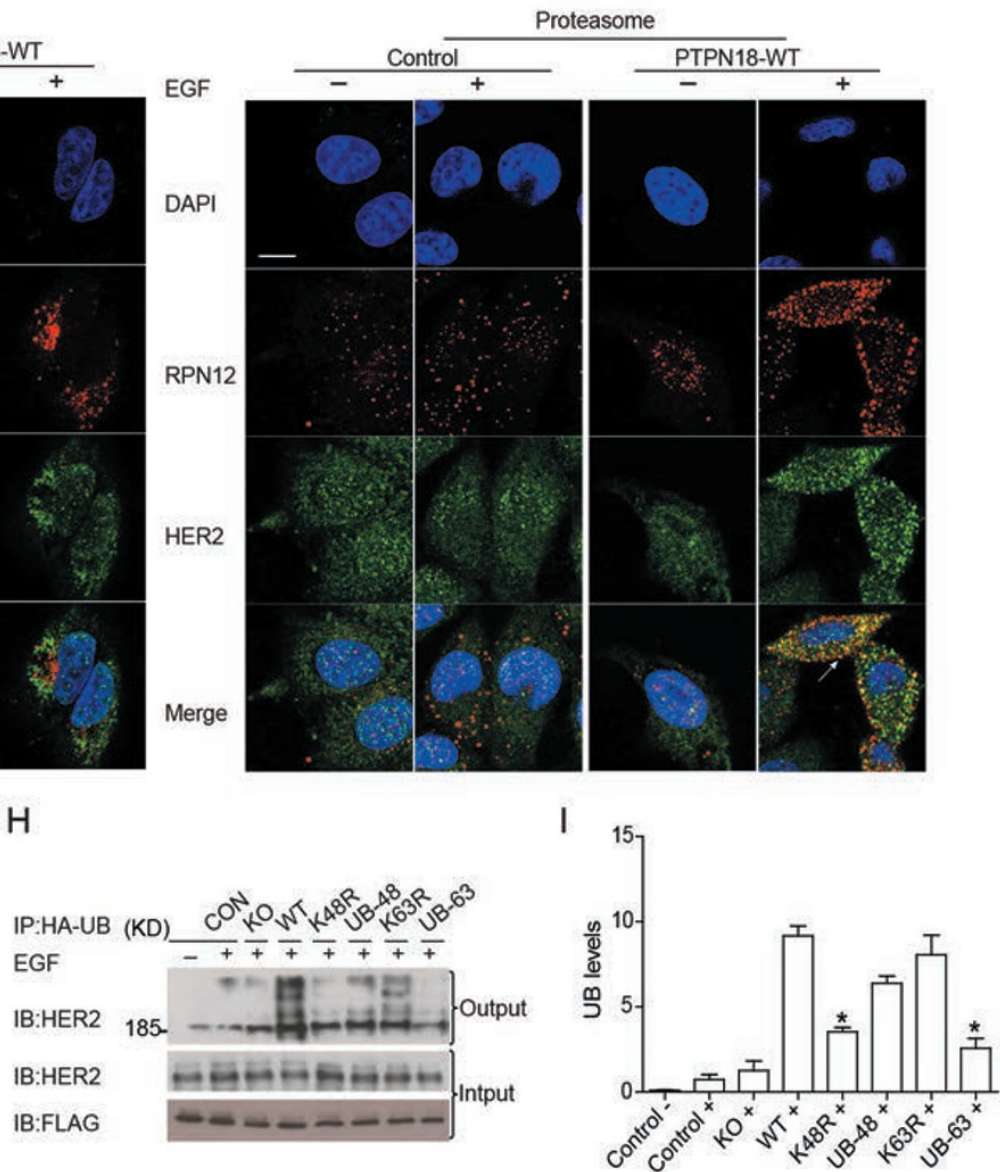

PTPN18 knockdown cells (Figure 9D and Supplementary information, Figure S9C). Conversely, the level of c-Cbl bound to HER2 and $\mathrm{pY}^{1112}$-phosphorylated HER2 decreased significantly with PTPN18-WT overexpression, but it increased with overexpression of the PTPN18
D197A dominant-negative mutant (Figure 9E and Supplementary information, Figure S9D). To determine the relation between the cellular outcome and the specific interaction between PTPN18 and the HER2 $\mathrm{Y}^{1112}$ site, based on the complex crystal structures of PTPN18 and the 
HER2 phospho-peptides, we then analyzed the binding of HER2 to c-Cbl in the biased PTPN18 mutants P109A and R140A (Figure 6 and Supplementary information, Figure S6). In agreement with biochemical and structural data, the P109A mutant weakened the effect of PTPN18 on HER2 $\mathrm{pY}^{1112}$ dephosphorylation and c-Cbl binding, whereas the R140A mutant had a similar effect to the wild type although its activity towards HER2 $\mathrm{pY}^{1248}$ and $\mathrm{pY}^{1196}$ sites was abolished (Figures 6A-6C and 9F and Supplementary information, Figure S9E). Therefore, the tyrosine phosphatase activity of PTPN18 and specific interactions between PTPN18 and the HER2 $\mathrm{Y}^{1112}$ site determined the HER2 $\mathrm{pY}^{1112}$ phosphorylation states and HER2 binding to c-Cbl. Taken together, these data suggest that PTPN18 decreases lysosome routing through its phosphatase activity and promotes proteasome targeting through its C-terminal PEST domain.

HER2 ubiquitination is regulated by the PTPN18/B-Trcp complex in the MCF-7 breast cancer cell line, and the HER2/PTPN18 ratio is correlated with breast cancer stage

Overexpression of HER2 has been highly correlated with breast cancer $[10,40]$. To investigate the PTPN18-regulated HER2 function in a more pathological context, we examined the expression of PTPN18 and HER2 in several cell lines derived from human breast cancers (Figure 10A and Supplementary information, Figure S10A). In all of these cell lines, both HER2 and PTPN18 were detected. SK-BR-3 cells have considerably higher HER2 expression but lower PTPN18 expression compared with other breast cancer cell lines, and the BT549, MDA-MB-436 and MCF-7 cell lines have relatively lower HER2 expression and higher PTPN18 expression.

We next chose the MCF-7 breast tumor cell line to examine the regulation of HER2 by the PTPN18/-Trcp complex because it has higher PTPN18 expression and detectable HER2 expression. In the MCF-7 cell line, PTPN18 forms a complex with $\beta$-Trcp, which was confirmed by co-immunoprecipitation of the endogenous PTPN18 and $\beta$-Trcp proteins (Figure 10B). Knockdown of either PTPN18 or $\beta$-Trcp decreased EGF-induced HER 2 ubiquitination, suggesting the regulation of HER2 ubiquitination by the PTPN18/ $\beta$-Trcp complex in MCF7 cells (Figure 10C-10E and Supplementary information, Figure S10B). Knockdown of either $\beta$-Trcp or $\mathrm{c}-\mathrm{Cbl}$ decreased but did not abolish EGF-induced HER2 ubiquitination. However, combined knockdown of $\beta$-Trcp and c-Cbl almost abolished EGF-induced HER2 ubiquitination (Figure 10D and 10E). These results indicate that $\beta$-Trcp and c-Cbl coordinately regulated HER2 ubiquitination. To dissect the functional role of PTPN18/ $\beta$-Trcp in the regulation of the HER2-mediated tumor cell motility in MCF-7 cells, we knocked down either PTPN18 or $\beta$-Trcp to monitor their effects on EGF-induced cell invasion. As a result, knockdown of either PTPN18 or $\beta$-Trcp expression significantly increased EGF-induced MCF-7 cell invasion (Figure 10F).

Finally, we examined the expression of HER 2 and PTPN18 in human breast tumors. The HER2 expression level had an increasing trend, whereas PTPN18 expression had a decreasing trend when comparing phase III

Figure 7 PTPN18 promoted K48-linked polyubiquitination and proteasomal degradation of HER2. (A) Knockdown of PTPN18, but not PTP-MEG2, reduced HER2 degradation under long-term EGF stimulation. Left: HER2 level was monitored by a specific antibody. Right: the amount of HER2 was quantified. ${ }^{*} P<0.05$, PTPN18-knockdown cells compared with control cells. (B) Overexpression of PTPN18, but not PTP-MEG2, enhanced HER2 degradation under long-term EGF stimulation. Left: HER2 levels were monitored by a specific antibody after stimulation with $100 \mathrm{ng} / \mathrm{ml}$ EGF. Right: the amount of HER2 was quantified. * $P<0.05$, PTPN18 transfected cells compared with control pCDNA3.1-transfected cells. (C) PTPN18 knockdown decreased HER2 ubiquitination. HepG2 cells were transfected with HA-ubiquitin. After $4 \mathrm{~h}$ of EGF stimulation, cells were lysed and ubiquitinated proteins were pulled down by anti-HA resin. Ubiquitinated HER2 was detected by anti-HER2 antibody. (D) Overexpression of PTPN18 enhanced HER2 ubiquitination. (E) Proteasome inhibitor MG-132 significantly decreased effects of PTPN18 on HER2 degradation. HepG2 cells were transfected with control vector (left upper) or PTPN18 (left lower) and preincubated with $5 \mu \mathrm{M} \mathrm{MG}-132$ for $16 \mathrm{~h}$ and $100 \mathrm{ng} / \mathrm{ml}$ EGF for the indicated time. The HER2 levels were monitored. Right: statistics. (F) Co-localization of the lysosome marker Lamp1 or proteasome marker RPN12 with HER2. Left: co-localization of HER2 with Lamp1, with or without PTPN18 overexpression, in EGF-treated or -untreated cells. Right: co-localization of HER2 with RPN12. HER2 was labeled with green, and the proteasome/lysosome marker was labeled with red. Overexpression of PTPN18 significantly increased the co-localization of HER2 with the proteasome marker RPN12. Scale bar, $10 \mu \mathrm{m}$. (G) Schematic representation of different ubiquitin mutations. KO: all seven Lys in the ubiquitin were mutated to Arg; UB-63 and UB-48: only Lys at site of 63 or 48, respectively, was not mutated to Arg; K48R and K63R: only Lys at site 48 or 63 , respectively, was mutated to Arg. $(\mathrm{H})$ The co-transfection of PTPN18 with different ubiquitin K-to-R mutants resulted in different levels of HER2 ubiquitination. PTPN18-WT and the HA-ubiquitin mutants displayed in $\mathbf{G}$ were transfected into HepG2 cells. The ubiquitinated HER2 was immunoprecipitated by anti-HA resin and detected by specific anti-HER2 antibody. (I) Statistics for $\mathbf{H}$. All statistics are from at least three independent experiments. ${ }^{*} P<0.05$, compared with control cells. 
A

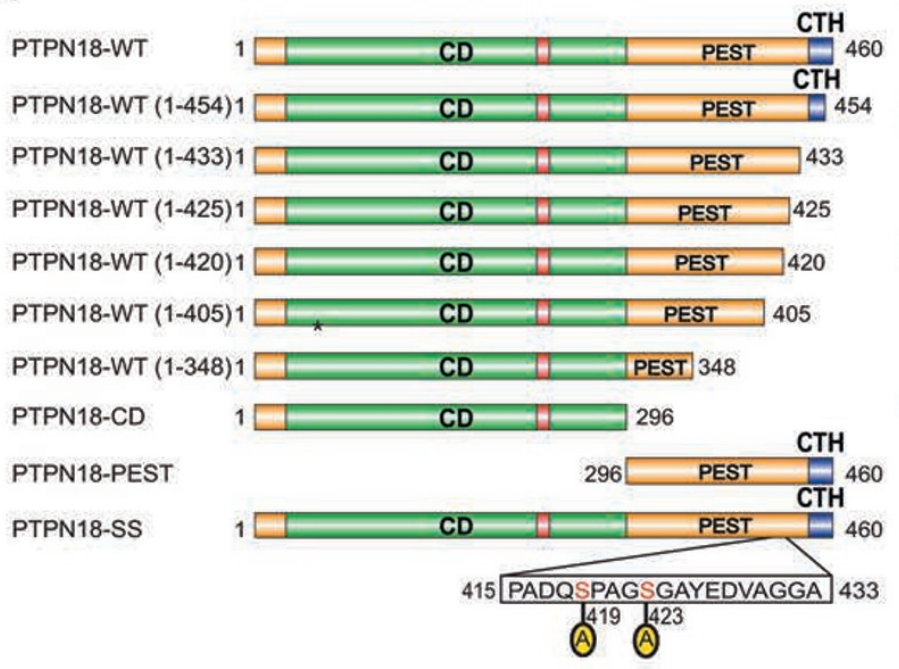

C

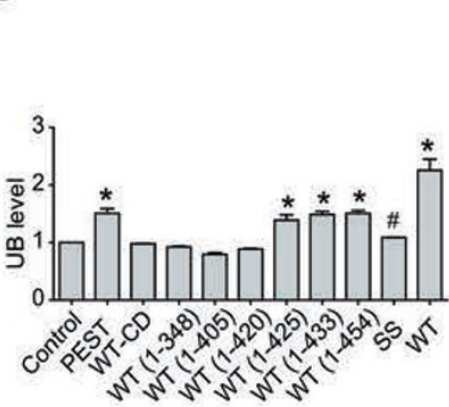

B

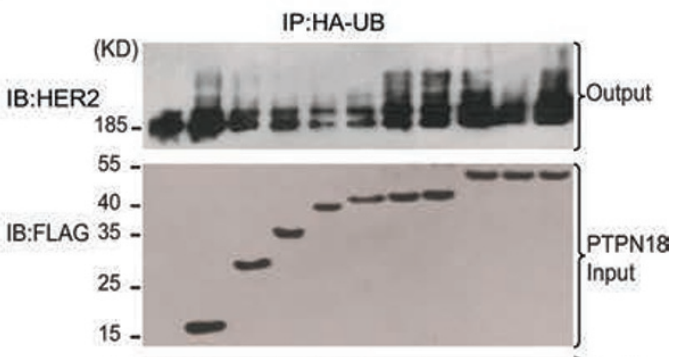

IB:HER2 185- PEPE-

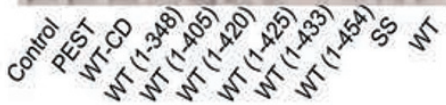

E

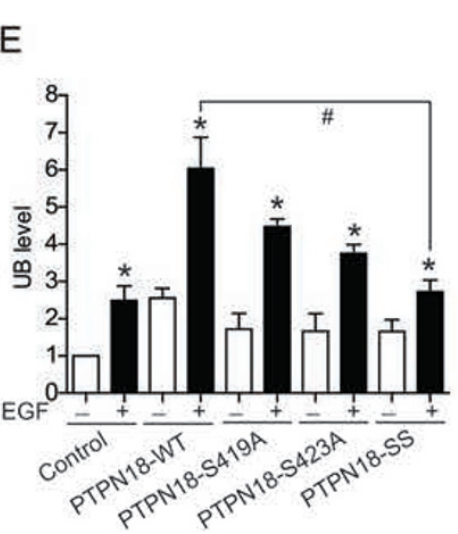

$\mathrm{F}$

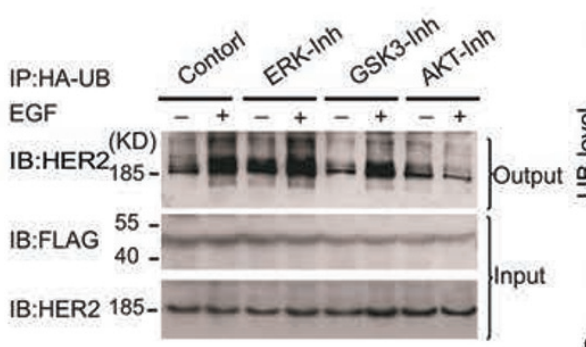

I

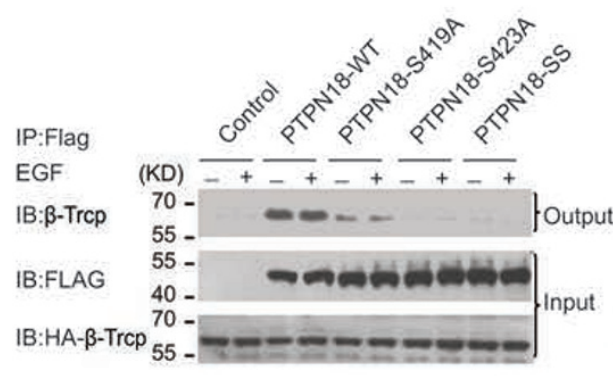

G

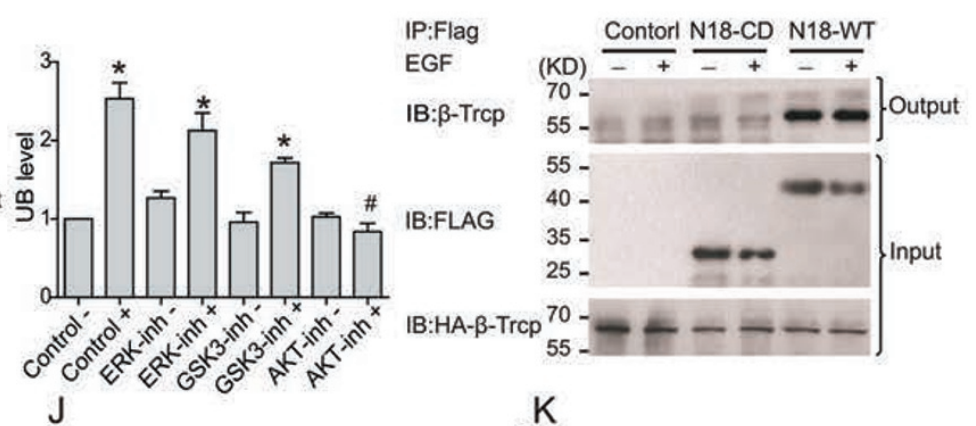

$\mathrm{H}$

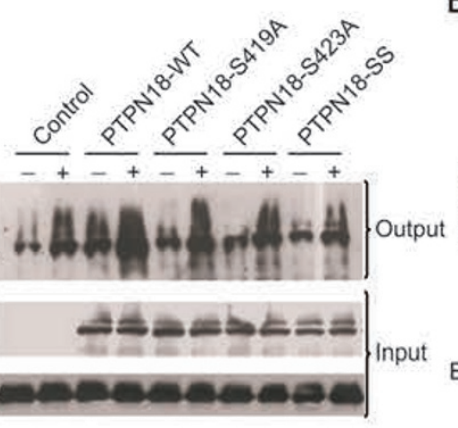

IB:HER2 18
$\mathrm{J}$

$\mathrm{K}$

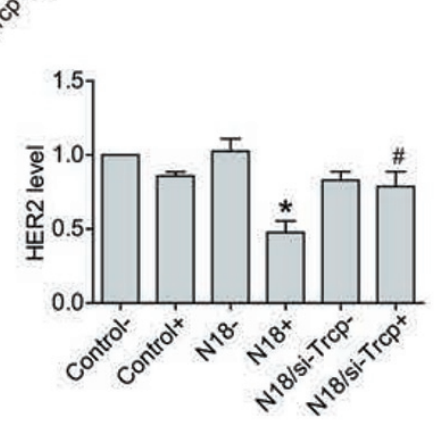


to phase II breast tumors (Supplementary information, Figure S10C). The HER2 or PTPN18 levels demonstrated no statistical significance when comparing phase III to phase II breast tumors, most likely due to the limited number of tumor samples available, but the HER2/ PTPN18 ratio was significantly higher in phase III compared with phase II breast tumors (Figure 10G and 10H). Taken together, these results show that PTPN18 and HER2 are co-expressed in many human breast cancer tissues and cell lines, and the HER2/PTPN18 ratio is correlated with breast cancer stage.

\section{Discussion}

Receptor activation is often accompanied by the phosphorylation and ubiquitination of multiple sites, which generates signaling barcodes to direct various downstream functions, as in the activation of $\beta 2$ adrenergic receptor and insulin receptor [41-43]. In the case of HER2, six tyrosine phosphorylation sites at the $\mathrm{C}$-terminus have been linked to various cellular outcomes and were shown to be precisely controlled by phosphorylation regulators, however, the precise mechanism was less well studied. In the present study, we demonstrated that PTPN18 specifically dephosphorylates HER2 $\mathrm{pY}^{1112}, \mathrm{pY}^{1196}$ and $\mathrm{pY}^{1248}$ sites among ten HER2 and EGFR C-terminal tyrosine phosphorylation sites (Figure 1). The crystal structures of PTPN18 in complex with three HER2 phospho-peptides revealed that two special structural features of the PTPN18 CD confer substrate recognition: (1) a specific hydrophobic $\mathrm{pY}+1$ site governing primary peptide-se- quence selectivity (Figure 4), and (2) the side-chain plasticity of R198 that is responsible for the recognition of two types of peptide substrate conformations (Figure 5). Furthermore, by examining the PTPN18 regulation of HER2 function via dephosphorylation of specific HER2 sites, we unexpectedly discovered that PEST domain of PTPN18 promotes HER2 ubiquitination and targets HER2 for proteasome degradation through K48-linked polyubiquitination and a phosphorylation-regulated mechanism (Figures 5 and 6). The "HER2-AKT-PTPN18/ $\beta$-Trcp-HER2 ubiquitination" feedback loop negatively regulates HER2 signaling. Our studies not only provide molecular details for the interaction between HER2 and PTPN18, but also reveal a novel mechanism of HER2 degradation and function of the PEST domain of PTP superfamily.

\section{Molecular mechanism of PTPN18-regulated HER2 phos- phorylation}

The catalytic domain of PTPN18 interacts with HER2 via a "kiss and run" mechanism and directs the recognition of the specific phosphorylated tyrosine sites of HER2 (Figure 1). In vitro, the catalytic domain of PTPN18 displays a strong activity toward HER2 $\mathrm{pY}^{1112}$, $\mathrm{pY}^{1196}$ and $\mathrm{pY}^{1248}$ phospho-peptides, but has a lower affinity toward other tested phospho-peptides and the small artificial substrate pNPP (Figure 2F, Supplementary information, Figure S2B-S2C and Table S1). The HER2:PTPN18 complex crystal structures revealed a hydrophobic pocket around the $\mathrm{pY}+1$ site, which is defined by the five residues V65, P272, A73, C233 and Q276.

Figure 8 Phosphorylated DQSPAGS motif in the PTPN18 PEST domain recruits E3 ligase $\beta$-Trcp and mediates HER2 ubiquitination. (A) Schematic representation of different PTPN18 truncations used in mapping the key motifs of PTPN18 in mediating HER2 ubiquitination. The C-terminal-specific motif containing S419 and S423 is highlighted. (B) The effects of different PTPN18 truncations on HER2 ubiquitination. (C) HER2 ubiquitination in B were quantified. ${ }^{*} P<0.05$, compared with control vectors. (D) Mutation effects of the potential phosphorylation sites located in the "DQSPAGS" motif on PTPN18-mediated HER2 ubiquitination. Flag-tagged PTPN18-WT, PTPN18-S419A, PTPN18-S423A or PTPN18-SS and HA-ubiquitin were co-transfected into HepG2 cells. After $100 \mathrm{ng} / \mathrm{ml}$ EGF stimulation, the ubiquitinated HER2 was immunoprecipitated and detected with a specific HER2 antibody. (E) HER2 ubiquitination in D was quantified. ${ }^{*} P<0.05$ compared with control cells. (F) Effects of ERK inhibitor, GSK3 inhibitor or AKT inhibitor on PTPN18-regulated HER2 ubiquitination. HepG2 cells expressing PTPN18 and HA-UB were pre-incubated with $100 \mathrm{nM}$ of the AKT inhibitor MK-22062. $\mathrm{HCl}$, or $15 \mu \mathrm{M}$ of the ERK inhibitor U0126 or $1 \mu \mathrm{M}$ of the GSK3 inhibitor CHIR99021 for $3 \mathrm{~h}$ and then stimulated with $100 \mathrm{ng} / \mathrm{ml} \mathrm{EGF}$. Ubiquitinated HER2 was monitored. (G) HER2 ubiquitination in $\mathbf{F}$ was quantified. ${ }^{*} P<0.05$ compared with non-EGF-stimulated cells. \#P $<$ 0.05 compared with control cells. $(\mathrm{H})$ The PEST domain is required for the specific interaction between PTPN18 and -Trcp. Flag-tagged full-length PTPN18 or its catalytic domain were co-transfected with HA- $\beta$-Trcp into HepG2 cells with or without EGF treatment. Formation of the PTPN18/ $\beta$-Trcp complex was detected by co-immunoprecipitation. (I) Mutation effects of the potential phosphorylation sites located in the "DQSPAGS" motif on specific $\beta$-Trcp interactions. The interactions between PTPN18-WT, PTPN18-S419A, PTPN18-S423A or PTPN18-SS (S419A/S423A) and $\beta$-Trcp were examined by co-immunoprecipitation. (J) Knockdown of $\beta$-Trcp reduced PTPN18-mediated HER2 degradation. HepG2 cells were transfected with control, PTPN18/SiRNA-control or PTPN18/SiRNA- $\beta$-Trcp. After EGF stimulation, HER2 and $\beta$-Trcp levels were detected by specific antibodies. (K) Statistics of $\mathbf{J}$. ${ }^{*} P<0.05$ compared with non-stimulated cells. $\# P<0.05$, siRNA- $\beta$-Trcp-treated cells compared with control-siRNA-treated cells. 
A

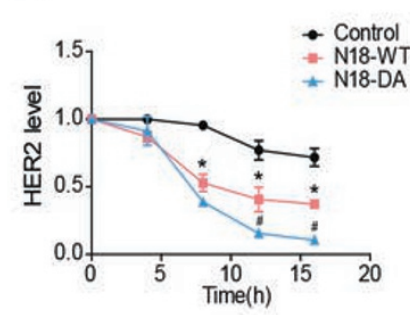

B

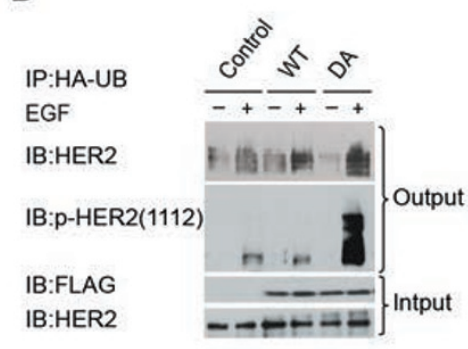

C

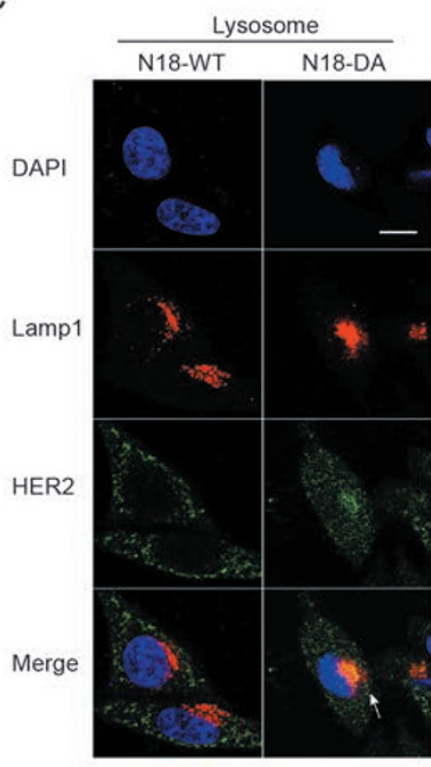

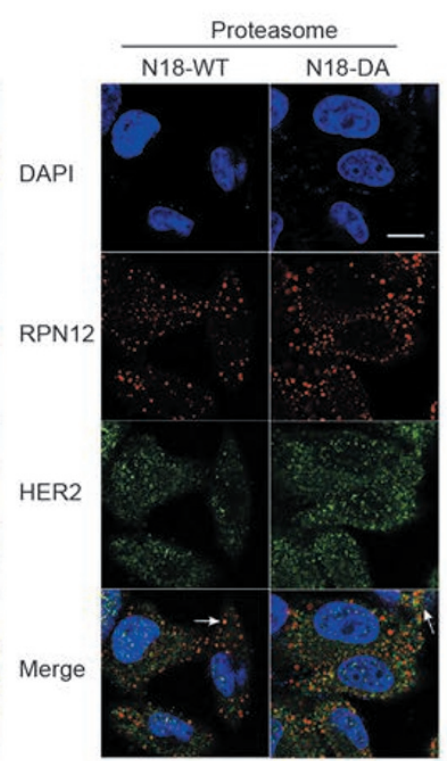

F

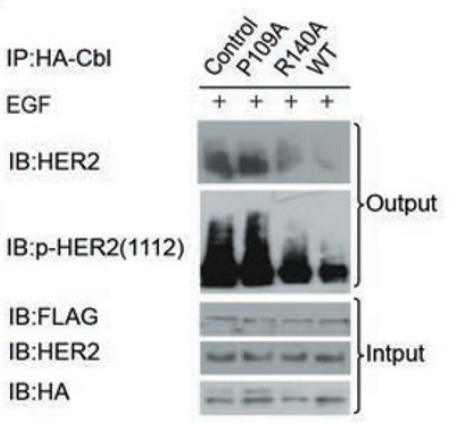

Figure 9 PTPN18 regulated the dynamics of HER2 degradation, and the dephosphorylation of HER2 pY ${ }^{1112}$ by PTPN18 functions as a switch between lysosome targeting and proteasome degradation. (A) The effect of the PTPN18 dominant-negative mutant D197A on HER2 degradation kinetics compared with PTPN18-WT. The data represent statistics from the densitometry quantification of the western blot (Supplementary information, Figure S9A). (B) The effect of the PTPN18-D197A on HER2 ubiquitination and phosphorylation at the $\mathrm{Y}^{1112}$ site. (C) Compared with the wild type, PTPN18-D197A enhanced HER2 lysosome localization but did not affect its localization to the proteasome. HER2, green; Lamp1 or RPN12, red. Scale bar, $10 \mu$ m. (D) Knockdown of PTPN18 by siRNA enhanced HER2/c-Cbl complex formation. HA-tagged c-Cbl was transfected into HepG2 cells. The HA-c-Cbl and HER2 complex was immunoprecipitated by anti-HA resin and detected with an anti-HER2 antibody. (E) PTPN18-D197A enhanced the binding of c-Cbl to HER2. HA-tagged c-Cbl and PTPN18-WT or D197A plasmids were transfected into HepG2 cells. The HA-c-Cbl and HER2 complex was detected as in D. (F) The effects of PTPN18 P109A or R140A mutations on the interaction of $\mathrm{c}-\mathrm{Cbl}$ and HER2. PTPN18-WT and R140A decreased HER2 $\mathrm{Y}^{1112}$ phosphorylation and the binding of $\mathrm{c}-\mathrm{Cb}$ to HER2, whereas the P109A mutant had much less effect. The level of c-Cbl-bound HER2 or phosphorylated HER2 at the $\mathrm{Y}^{1112}$ site in the immune complexes was detected with specific antibodies.

Except for Q276 of PTPN18, these residues in the hydrophobic pocket are not conserved, and the combination of these four residues is unique among all known HER2 tyrosine phosphatases (Figure 6A and Supplementary information, Figure S6). Mutations of these residues to corresponding PTP proteins (e.g., V65I and P272M) significantly reduce the activity of PTPN18 toward HER2 $\mathrm{pY}^{1196}$ and $\mathrm{pY}^{1248}$ phospho-peptides. Therefore, the hy- drophobic pocket surrounding the $\mathrm{pY}+1$ site is one of the determinants for PTPN18 substrate specificity.

Residues from other loops also contribute to substrate specificity of PTPN18. The unique R198 in the WPD loop, the combination of the F30 and Q34 in the $\alpha 1$ helix and the combination of the " ${ }^{\prime 138} \mathrm{RKR}^{140 "}$ in $\beta 6$ are all unique for PTPN18. Mutations of R198, Q34, R138 and R140 to Ala all decreased activity of PTPN18 toward 
specific HER2 phospho-peptides (Figure 6A-6B and Supplementary information, Figure S6). In particular, the side-chain plasticity of R198 in the WPD loop is responsible for two different phospho-peptide binding modes. The R198 in the WPD loop mediating PTPN18 substrate plasticity is reminiscent of another R47 in the pY loop of PTP1B, which assumes different conformations upon binding to acidic or hydrophobic residues $[33,34]$. Although presenting on different loops and in different surface positions, the combined information derived from these two sets of structures indicates that substrate selectivity governed by the plasticity of the surface Arg may be an important mechanism for the substrate recognition of PTPs.

The three complex structures also revealed different interactions between PTPN18-HER2 $\mathrm{pY}^{1196} / \mathrm{HER} 2 \mathrm{pY}^{1248}$ and PTPN18-HER2 $\mathrm{pY}^{1112}$. Whereas P109 of PTPN18 specifically interacts with HER2 $\mathrm{pY}^{1112}$, R140 of PTPN18 specifically interacts with HER2 $\mathrm{pY}^{1196}$ and HER2 $\mathrm{pY}^{1248}$. Utilizing biased mutants derived from the crystallographic and enzymology studies, we demonstrated that specific disruption of the PTPN18:HER2 $\mathrm{pY}^{1196} / \mathrm{HER} 2 \mathrm{pY}^{1248}$ interface, but not the PTPN18:HER2 $\mathrm{pY}^{1112}$ interface, altered phosphorylation network downstream of HER2 for invasion and proliferation (Figure 6C-6E). Conversely, specific disruption of the PTPN18:HER2 $\mathrm{pY}^{1112}$ interface abolished the activity of PTPN18 in the regulation of the HER 2/c-Cbl interaction and HER2 degradation dynamics (Figure 9)

In addition to the specific structural features in the CD of PTPN18, which substantially contributes to the specific PTPN18-HER2 interaction and regulation of the HER2 function, it is worth noting that the C-terminal PEST domain of PTPN18 is another important determinant for HER2 recognition (Figure 2D-2E). The current structures only reveal information for the CD of PTPN18 and a minimal region surrounding the specific HER2 phosphorylation sites. Future structural studies with fulllength PTPN18 and HER2 are required to elucidate the exact mechanism of regulation of HER2 by PTPN18.

\section{Molecular mechanism of PTPN18-regulated HER2 ubiq- uitination}

Previous studies have revealed that HER2 weakly associates with $\mathrm{c}-\mathrm{Cbl}$ for lysosome routing after EGF stimulation [21, 44]. Alternatively, HER2 might be ubiquitinated and transported to the proteasome in certain cellular contexts, such as following the application of the HSP90 chaperone inhibitor geldanamycin [22]. Compared with the rapid degradation of HER2 by the proteasome, c-Cbl-mediated HER2 degradation is slower. This continual HER2 receptor trafficking in cells might result in returning of more receptors to the cell surface or assume other signaling functions [45]. Here, we discovered that PTPN18 specifically deregulates the HER2 $\mathrm{pY}^{1112}$ phosphorylation state and blocks the interaction between HER2 and c-Cbl, resulting in a decrease in lysosome routing. Unexpectedly, PTPN18 also promoted the distribution of HER2 to the proteasome for rapid HER2 destruction, suggesting fine-tuning of receptor ubiquitination barcode in parallel with the phosphorylation barcode. Therefore, PTPN18 functions as a switch from lysosome routing and cellular trafficking to rapid proteasomal destruction of HER2. The functional importance of this shift could be better understood with further studies on c-Cbl-mediated HER2 subcellular trafficking and the function of K63-linked HER2 ubiquitination.

\section{Novel function of the C-terminal regulatory PEST do- main}

In addition to the catalytic region, the majority of the PTP family members contain either regulatory motifs or domains. The functional roles of the $\mathrm{SH} 2$ domain in SHPs and the KIM domain in STEP or HePTP are demonstrated to be important for their subcellular localization and binding to substrates; however, the roles of the regulatory domains in most other PTPs have not been elucidated [46-50]. In the case of PEST domain-containing phosphatases, including PTPN12, PTPN18 and PTPN22, the PEST domains are reported to mediate their substrate binding, but not related to their own ubiquitiantion or degradation. Here, we report a new functional role of the PEST domain of PTPN18, which recruits the E3 ligase $\beta$-Trcp for HER2 ubiquitination (Figure 8). PTPN18-mediated HER2 degradation is primarily K48linked, and shifts HER2 from the slower lysosome-dependent degradation to the more rapid proteasome degradation (Figure 9). Therefore, our results reveal a previously unidentified mechanism for the regulatory domain of tyrosine phosphatases.

The PTPN18-dependent HER2 degradation is also reminiscent of Vpu-mediated CD4 receptor degradation [51]. In both cases, phosphorylation occurs on the ancillary protein (Vpu or PTPN18) rather than the receptor (CD4 or HER2). Furthermore, PTPN18 associates with CSK and PSTPIP, and members of the small PTP-PEST family (PTPN12, PTPN18 and PTPN22) all contain PEST domains at their C-termini. Both PTPN12 and PTPN22 are important signal transducers and are highly related to human diseases, such as cancer, diabetes and other autoimmune diseases [24, 29, 52]. Whether PTPN18 also promotes CSK and PSTPIP ubiquitination and whether all PEST-family phosphatases regulate ubiquitination of associated proteins in a phosphorylation-de- 
A

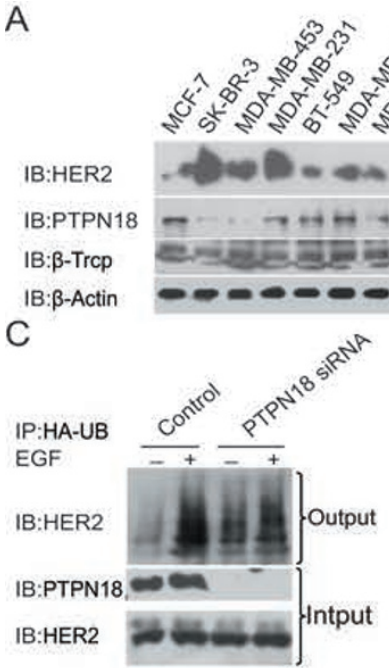

B

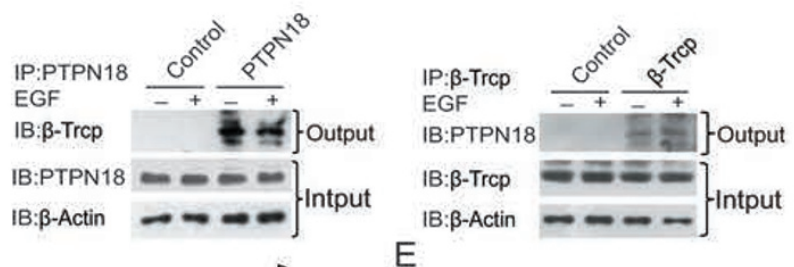

D

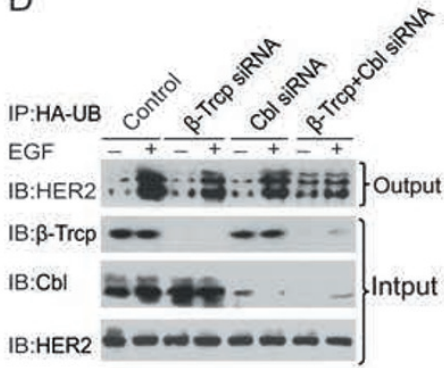

$\mathrm{F}$

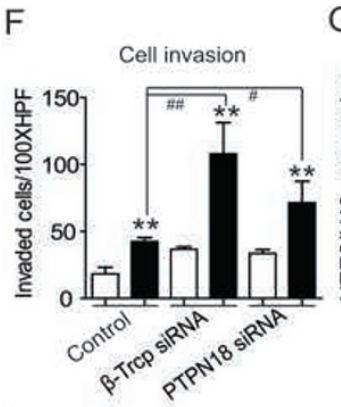

G
$\mathrm{H}$

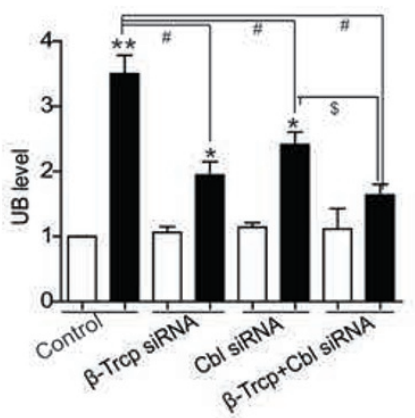

I

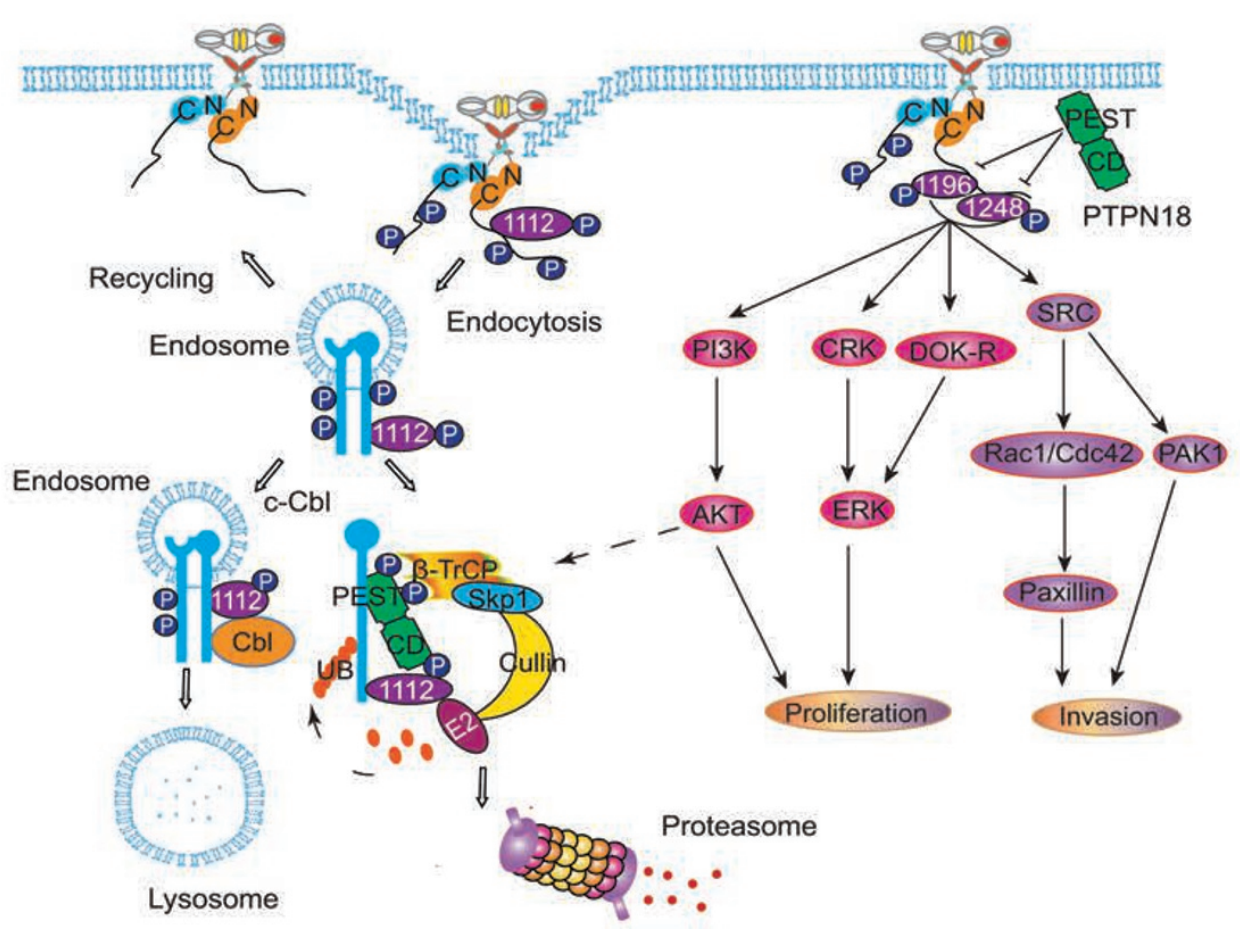


pendent manner are worthy of further investigation.

\section{Materials and Methods}

\section{Phosphatase assays}

pNPP (Bio Basic) was used in enzymatic reactions to determine the intrinsic catalytic activity of PTPN18 and its mutants. All enzymatic reactions using pNPP substrate were carried out in DMG buffer (50 mM 3,3-dimethyl glutarate, $\mathrm{pH}$ 7.0, 1 mM EDTA, 1 $\mathrm{mM}$ DTT) and the ionic strength was maintained at $0.15 \mathrm{M}$ (adjusted by the addition of $\mathrm{NaCl}$ ). Assays were initiated by adding enzyme to the reaction mixtures with substrate at different concentrations. Reactions were carried out in microplates at $37^{\circ} \mathrm{C}$. The dephosphorylation of pNPP was terminated by adding $120 \mu 1$ of 1 $\mathrm{M} \mathrm{NaOH}$, and the enzymatic activity was monitored by measuring the absorbance at $405 \mathrm{~nm}$ [28]. Kinetic parameters were determined using the nonlinear regression hyperbolic fit to the Michaelis-Menten equation.

The PTPN18-catalyzed hydrolysis of pY-containing peptides was assayed by two different methods. The first was monitoring at $305 \mathrm{~nm}$ with excitation at $280 \mathrm{~nm}$, showing the increase in tyrosine fluorescence after dephosphorylation [53]. The second method was an inorganic-phosphate assay with the end-point reading at 620 $\mathrm{nm}$, as previously described [54]. The reaction was carried out at $37^{\circ} \mathrm{C}$ in $50 \mathrm{mM}$ 3,3-dimethylglutarate buffer $(\mathrm{pH} 6.5)$ containing $1 \mathrm{mM}$ DTT and $1 \mathrm{mM}$ EDTA with an ionic strength of $0.15 \mathrm{M}$. All reactions were initiated by adding PTPN18 to a final concentration of $10 \mathrm{nM}$. The data were analyzed using nonlinear least-squares regression (KaleidaGraph, Synergy Software).

\section{Structural determination and refinement}

The crystals of PTPN18-HER2-pY $Y^{1248}$ and -pY ${ }^{1112}$ peptides belong to the $\mathrm{P} 22_{1}$ space group, and the crystal of the PTPN18-HER2-
$\mathrm{pY}{ }^{1196}$ peptide belongs to the $\mathrm{P} 22_{1} 2_{1} 2_{1}$ space group. In each asymmetric unit, the PTPN18-HER2-pY ${ }^{1248}$ contains one monomer, the PTPN18-HER2-pY ${ }^{1196}$ contains a dimer and the PTPN18-HER2$\mathrm{pY}^{1112}$ peptide complex contains a tetramer. The structures of PTPN18-HER2-pY ${ }^{1248}, \mathrm{pY}^{1112}$ and $\mathrm{pY}^{1196}$ were determined by molecular replacement with Phaser [55] in the CCP4 software package. A single chain of the PTPN18 catalytic domain (PDB code: 2OC3, water deleted) was used as the initial search model [56, 57]. Further refinements were carried out using the PHENIX program with iterative manual building in COOT [57]. Composite omitted maps were built, and density modification was carried out to reduce the phase biases during further refinement. Ramachandran plots were calculated using PROCHECK and COOT. The statistics for the final solved structures are shown in Supplementary information, Table S2.

\section{GST pull-down}

Plasmids expressing GST or GST fusion proteins were transformed into E. coli BL21. The bacteria were grown for $3 \mathrm{~h}$ at 37 ${ }^{\circ} \mathrm{C}$, then incubated with $0.3 \mathrm{mM}$ isopropyl-1-thio- $\beta$-D-galactopyranoside for $12 \mathrm{~h}$ at $25^{\circ} \mathrm{C}$. The bacteria were harvested and lysed by sonication. Then, GST and GST fusion proteins were incubated with glutathione-Sepharose 4B (Amersham Biosciences) for $2 \mathrm{~h}$ at $4{ }^{\circ} \mathrm{C}$ followed by extensively washing with PBS containing $1 \%$ Triton. The GST fusion proteins were then eluted by glutathione, and the purity of the proteins was examined by electrophoresis. Purified GST or GST fusion proteins were immobilized on glutathione-Sepharose 4B beads and then incubated with cell lysates (3 mg) overnight at $4{ }^{\circ} \mathrm{C}$ with gentle agitation. The beads were collected by centrifugation and washed eight times with cell lysis buffer. After removing the supernatant in the final wash, the samples were re-suspended in $2 \times$ SDS loading buffer and boiled for $5 \mathrm{~min}$, and the proteins retained on the beads were analysed by western

Figure 10 HER2 ubiquitination is regulated by the PTPN18/ $\beta$-Trcp complex in breast cancer cell lines and the HER2/PTPN18 ratio (expression ratio) is correlated with breast cancer stage. (A) Protein expression levels of PTPN18 and HER2 in seven breast cancer cell lines and HepG2. (B) Endogenous interaction between PTPN18 and $\beta$-Trcp was revealed by co-immunoprecipitation in MCF-7 cells. Left: PTPN18 was immunoprecipitated with protein $A+G$ agarose beads and PTPN18 antibody with or without $100 \mathrm{ng} / \mathrm{ml}$ EGF stimulation for $30 \mathrm{~min}$. PTPN18-associated $\beta$-Trcp was detected with a specific $\beta$-Trcp antibody. Right: reverse immunoprecipitation of PTPN18 with the $\beta$-Trcp antibody. (C) MCF-7 cells were transfected with HA-ubiquitin and PTPN18 siRNA. After $48 \mathrm{~h}$, cells were stimulated with $100 \mathrm{ng} / \mathrm{ml}$ EGF for $4 \mathrm{~h}$. Ubiquitinated HER2 was immunoprecipitated by anti-HA resin and detected with an anti-HER2 antibody. (D) Coordinated regulation of EGF-induced HER2 ubiquitination by $\beta$-Trcp and c-Cbl. MCF-7 cells were transfected with HA-ubiquitin and siRNAs directed against $\beta-$ Trcp or c-Cbl. Ubiquitinated HER2 was examined with an anti-HER2 antibody as in C. (E) The relative ubiquitinated HER2 level in $\mathbf{D}$ was quantified and shown as a bar graph. ${ }^{*} P<0.05,{ }^{* *} P<0.01$ compared with non-EGF-stimulated cells. $\# P<$ 0.05 compared with control siRNA. $\$ P<0.05$ compared with Cbl siRNA. (F) Effects of siRNA-mediated knockdown of the $\beta$-Trcp expression on EGF-induced MCF-7 cell invasion. All statistics are from at least three independent experiments. ${ }^{*} P<$ 0.05 compared with non-EGF-stimulated cells. \#P<0.05 compared with control. (G, H) The expression ratio of HER2 over PTPN18 (HER2/PTPN18) was significantly increased in tissues from breast cancer patients with stage III tumors compared with tissues from breast cancer patients with stage II tumors. (G), Relative HER2 expression divided by PTPN18 expression (See Supplementary information, Figure S10 for original western blots). (H) Statistics for G. (I) A proposed working model of PTPN18 regulated HER2 tyrosine phosphorylation and ubiquitination pathway. After activation, HER2 was phosphorylated on multiple sites at its C-terminus. PTPN18 specifically dephosphorylated HER2 $\mathrm{pY}^{1196}$ and $\mathrm{pY} \mathrm{Y}^{1248}$ sites and inhibited HER2 signaling cascade for cell proliferation and invasion. Meanwhile, PTPN18 not only blocked the c-Cbl-mediated lysosome targeting of HER2 through dephosphorylation of HER2 on $\mathrm{pY}^{1112}$ site, but also recruited E3 ligase $\beta$-Trcp for K48-linked HER2 ubiquitination and proteasomal degradation. The interaction between PTPN18 and $\beta$-Trcp is phosphorylation regulated and requires AKT activation. The "HER2-AKT-PTPN18-HER2 ubiquitination" is a negative feedback loop for HER2 regulation. 
blotting.

\section{Cell invasion assay}

Twenty-four hours after transfection, HepG2 cells were resuspended in DMEM (serum free) and added $\left(1.25 \times 10^{5} / 100 \mu \mathrm{l}\right)$ to each Matrigel-coated upper chamber (BD Bioscience). The lower chamber contained $15 \mathrm{ng} / \mathrm{ml} \mathrm{EGF}$ as a chemoattractant. After 24 $\mathrm{h}$, un-migrated cells were removed and the inserts were stained with methanol and crystal violet. Then, eight randomly selected high-power fields per insert were used to quantify the number of migrated cells. Each experiment was repeated at least in triplicate. Data analysis was conducted with GraphPad software.

\section{MTT assay}

Cells transfected with control vectors, PTP-MEG2, wild-type PTPN18, or different PTPN18 mutants were seeded in 96-well plates containing DMEM and 10\% FBS. Cell proliferation was then determined by MTT assay over a $36-\mathrm{h}$ period by reading $\mathrm{OD}_{450}$ through a multi-well spectrophotometer (Molecular Device $190 ; 15)$. A standard curve was obtained by plotting cell numbers against absorbance values.

\section{Detection of ubiquitinated HER2}

HepG2 cells were co-transfected with HA-ubiquitin and various PTPN18 constructs to assess the effects of different PTPN18 domains on EGF-induced HER2 ubiquitination. Two days after transfection, the cells were treated with $100 \mathrm{ng} / \mathrm{ml} \mathrm{EGF}$ or medium for $6 \mathrm{~h}$ and a proteasome inhibitor (MG132, $10 \mathrm{~nm}$ ) was added. The cells were then scraped and resuspended in lysis buffer $(1 \%$ Triton $\mathrm{X}-100,150 \mathrm{mM} \mathrm{NaCl}, 25 \mathrm{mM}$ HEPES, $10 \mathrm{mM} \mathrm{MgCl}_{2}, 1 \mathrm{mM}$ EDTA and 2\% glycerol) [58]. After $1 \mathrm{~h}$ of end-over-end rotation at $4{ }^{\circ} \mathrm{C}$, the lysate was cleared by high-speed centrifugation. The soluble lysates were incubated with $30 \mu \mathrm{l}$ HA affinity beads for 2 $\mathrm{h}$. The beads were washed at least three times with lysis buffer and then resuspended in $60 \mu 1$ of $2 \times$ sample buffer. The ubiquitinated HER2 was detected by specific antibody. Different ubiquitin mutations on specific lysines (K48, K63, R48, R63 and KO) were also created and tested for specific HER2 polyubiquitination.

\section{Co-immunoprecipitation}

HepG2 cells were cultured in 10-cm plates and were transiently transfected with pcDNA3.1-FLAG control plasmid or plasmids encoding different PTPN18 fragments. Two days after transfection, the cells were treated with $100 \mathrm{ng} / \mathrm{ml} \mathrm{EGF}$ at certain time points. The cell lysates were subjected to immunoprecipitation by incubating with anti-FLAG-beads overnight at $4{ }^{\circ} \mathrm{C}$. Immune complexes were analyzed by western blotting with specific antibodies. For c-Cbl and HER2 interaction, the HA-c-Cbl was immunoprecipitated by HA-affinity agarose and the associated HER2 was detected by specific antibodies.

\section{Immunofluorescence}

Cells seeded in 24-well plates on chamber slides were cultured overnight, washed with PBS and fixed with 4\% PFA for $10 \mathrm{~min}$ at room temperature. After cell permeabilization in $0.5 \%$ Triton $\mathrm{X}-100$ for $10 \mathrm{~min}$, cells were blocked with 5\% normal goat serum for $30 \mathrm{~min}$ at room temperature, then incubated with primary antibody ( $2.5 \%$ serum dilution) overnight. Secondary antibodies were goat anti-mouse Alexa Fluor 488 and anti-rabbit Alexa Fluor 555 (Invitrogen). Cells were incubated with secondary antibody for 1 h. Slides were mounted in Prolong Gold (Invitrogen), stained with 4',6-diamidino-2-phenylindole and left in dark before fluorescence microscopic analysis.

\section{Accession codes}

Coordinates and structural factors were deposited in the Protein Data Bank with the following accession codes: 4GFU (PTPN18HER2-pY ${ }^{1248}$ phospho-peptide), 4GFV (PTPN18-HER2-pY ${ }^{1196}$ phospho-peptide), and 4NND (PTPN18-HER2-pY 1112 phospho-peptide).

(All other reagents and methods are described in Supplementary information, Data $\mathrm{S} 1$.)

\section{Acknowledgments}

This work was supported by the National Key Basic Research Program of China (2013CB967700 to X Yu; 2012CB910402 to JP Sun), the National Natural Science Foundation of China (31000362 to X Yu, 31100580 and 31271505 to JP Sun, and 81171062 to Q Pang), and the Shandong Natural Science Fund for Distinguished Young Scholars (JQ201320 to X Yu), the Fundamental Research Funds of Shandong University (2014JC029 to X Yu), the State Key Laboratory of Cell Biology and the Program for Changjiang Scholars and Innovative Research Team in University (IRT13028).

\section{References}

1 Levi-Montalcini R, Cohen S. Effects of the extract of the mouse submaxillary salivary glands on the sympathetic system of mammals. Ann N Y Acad Sci 1960; 85:324-341.

2 Cohen S, Levi-Montalcini R. Purification and properties of a nerve growth-promoting factor isolated from mouse sarcoma 180. Cancer Res 1957; 17:15-20.

3 Cohen $\mathrm{S}$. The stimulation of epidermal proliferation by a specific protein (EGF). Dev Biol 1965; 12:394-407.

4 Sharma SV, Bell DW, Settleman J, Haber DA. Epidermal growth factor receptor mutations in lung cancer. Nat Rev Cancer 2007; 7:169-181.

5 Eckhart W, Hutchinson MA, Hunter T. An activity phosphorylating tyrosine in polyoma T antigen immunoprecipitates. Cell 1979; 18:925-933.

6 Ushiro H. Identification of phosphotyrosine as a product of epidermal growth factor-activated protein kinase in A-431 cell membranes. J Biol Chem 1980; 255:8363-8365.

7 Kasuga M, Zick Y, Blithe DL, Crettaz M, Kahn CR. Insulin stimulates tyrosine phosphorylation of the insulin receptor in a cell-free system. Nature 1982; 298:667-669.

8 Kasuga M, Karlsson FA, Kahn CR. Insulin stimulates the phosphorylation of the 95,000-dalton subunit of its own receptor. Science 1982; 215:185-187.

9 Gschwind A, Fischer OM, Ullrich A. The discovery of receptor tyrosine kinases: targets for cancer therapy. Nat Rev Cancer 2004; 4:361-370.

10 Sachdev JC, Jahanzeb M. Blockade of the HER family of receptors in the treatment of HER2-positive metastatic breast cancer. Clin Breast Cancer 2012; 12:19-29.

11 Zhang X, Gureasko J, Shen K, Cole PA, Kuriyan J. An allosteric mechanism for activation of the kinase domain of epidermal growth factor receptor. Cell 2006; 125:1137-1149. 
12 Lucs AV, Muller WJ, Muthuswamy SK. She is required for ErbB2-induced inhibition of apoptosis but is dispensable for cell proliferation and disruption of cell polarity. Oncogene 2010; 29:174-187.

13 Marone R, Hess D, Dankort D, Muller WJ, Hynes NE, Badache A. Memo mediates ErbB2-driven cell motility. Nat Cell Biol 2004; 6:515-522.

14 Ren Z, Schaefer TS. ErbB-2 activates Stat3 alpha in a Srcand JAK2-dependent manner. J Biol Chem 2002; 277:3848638493.

15 Dankort D, Maslikowski B, Warner N, et al. Grb2 and Shc adapter proteins play distinct roles in Neu (ErbB-2)-induced mammary tumorigenesis: implications for human breast cancer. Mol Cell Biol 2001; 21:1540-1551.

16 Dankort D, Jeyabalan N, Jones N, Dumont DJ, Muller WJ. Multiple ErbB-2/Neu phosphorylation sites mediate transformation through distinct effector proteins. J Biol Chem 2001; 276:38921-38928.

17 Jones RB, Gordus A, Krall JA, MacBeath G. A quantitative protein interaction network for the ErbB receptors using protein microarrays. 2006; Nature 439:168-174.

18 Cicenas J, Urban P, Kung W, et al. Phosphorylation of tyrosine 1248-ERBB2 measured by chemiluminescence-linked immunoassay is an independent predictor of poor prognosis in primary breast cancer patients. Eur J Cancer 2006; 42:636645.

19 Taniyama K, Ishida K, Toda T, et al. Tyrosine1248-phosphorylated HER2 expression and HER2 gene amplification in female invasive ductal carcinomas. Breast Cancer 2008; 15 : 231-240.

20 Bourguignon LY, Zhu H, Zhou B, Diedrich F, Singleton PA, Hung MC. Hyaluronan promotes CD44v3-Vav2 interaction with Grb2-p185(HER2) and induces Rac1 and Ras signaling during ovarian tumor cell migration and growth. J Biol Chem 2001; 276:48679-48692.

21 Klapper LN, Waterman H, Sela M, Yarden Y. Tumor-inhibitory antibodies to HER-2/ErbB-2 may act by recruiting c-Cbl and enhancing ubiquitination of HER-2. Can Res 2000; 60:3384-3388.

$22 \mathrm{Xu} \mathrm{W,} \mathrm{Marcu} \mathrm{M,} \mathrm{Yuan} \mathrm{X,} \mathrm{Mimnaugh} \mathrm{E,} \mathrm{Patterson} \mathrm{C,} \mathrm{Neckers}$ L. Chaperone-dependent E3 ubiquitin ligase CHIP mediates a degradative pathway for c-ErbB2/Neu. Proc Natl Acad Sci USA 2002; 99:12847-12852.

23 Gensler M, Buschbeck M, Ullrich A. Negative regulation of HER2 signaling by the PEST-type protein-tyrosine phosphatase BDP1. J Biol Chem 2004; 279:12110-12116.

24 Sun T, Aceto N, Meerbrey KL, et al. Activation of multiple proto-oncogenic tyrosine kinases in breast cancer via loss of the PTPN12 phosphatase. Cell 2011; 144:703-718.

25 Yuan T, Wang Y, Zhao ZJ, Gu H. Protein-tyrosine phosphatase PTPN9 negatively regulates ErbB2 and epidermal growth factor receptor signaling in breast cancer cells. $\mathrm{J}$ Biol Chem 2010; 285:14861-14870.

26 Zhu JH, Chen R, Yi W, et al. Protein tyrosine phosphatase PTPN13 negatively regulates Her2/ErbB2 malignant signaling. Oncogene 2008; 27:2525-2531.

27 Veillette A, Rhee I, Souza CM, Davidson D. PEST family phosphatases in immunity, autoimmunity, and autoinflammatory disorders. Immunol Rev 2009; 228:312-324.
28 Yu X, Sun JP, He Y, et al. Structure, inhibitor, and regulatory mechanism of Lyp, a lymphoid-specific tyrosine phosphatase implicated in autoimmune diseases. Proc Natl Acad Sci USA 2007; 104:19767-19772.

29 Bottini N, Musumeci L, Alonso A, et al. A functional variant of lymphoid tyrosine phosphatase is associated with type I diabetes. Nat Genet 2004; 36:337-338.

$30 \mathrm{Yu}$ X, Chen M, Zhang S, et al. Substrate specificity of lymphoid-specific tyrosine phosphatase (Lyp) and identification of Src kinase-associated protein of $55 \mathrm{kDa}$ homolog (SKAPHOM) as a Lyp substrate. J Biol Chem 2011; 286:3052630534.

31 Rhee I, Veillette A. Protein tyrosine phosphatases in lymphocyte activation and autoimmunity. Nat Immunol 2012; 13:439447.

32 Salmeen A, Andersen JN, Myers MP, Tonks NK, Barford D. Molecular basis for the dephosphorylation of the activation segment of the insulin receptor by protein tyrosine phosphatase 1B. Mol Cell 2000; 6:1401-1412.

33 Sarmiento M, Puius YA, Vetter SW, et al. Structural basis of plasticity in protein tyrosine phosphatase 1B substrate recognition. Biochemistry 2000; 39: 8171-8179.

34 Sun JP, Fedorov AA, Lee SY, et al. Crystal structure of PTP1B complexed with a potent and selective bidentate inhibitor. J Biol Chem 2003; 278:12406-12414.

35 Puius YA, Zhao Y, Sullivan M, Lawrence DS, Almo SC, Zhang ZY. Identification of a second aryl phosphate-binding site in protein-tyrosine phosphatase 1B: a paradigm for inhibitor design. Proc Natl Acad Sci USA 1997; 94:13420-13425.

36 Yarden Y, Sliwkowski MX. Untangling the ErbB signalling network. Nat Rev Mol Cell Biol 2001; 2:127-137.

37 Huang F, Kirkpatrick D, Jiang X, Gygi S, Sorkin A. Differential regulation of EGF receptor internalization and degradation by multiubiquitination within the kinase domain. Mol Cell 2006; 21:737-748.

38 Meyer RD, Srinivasan S, Singh AJ, Mahoney JE, Gharahassanlou KR, Rahimi N. PEST motif serine and tyrosine phosphorylation controls vascular endothelial growth factor receptor 2 stability and downregulation. Mol Cell Biol 2011; 31:2010-2025.

39 Frescas D, Pagano M. Deregulated proteolysis by the F-box proteins SKP2 and beta-TrCP: tipping the scales of cancer. Nat Rev Cancer 2008; 8:438-449.

40 Arteaga CL, Sliwkowski MX, Osborne CK, Perez EA, Puglisi F, Gianni L. Treatment of HER2-positive breast cancer: current status and future perspectives. Nat Rev Clin Oncol 2012; 9:16-32.

41 Nobles KN, Xiao K, Ahn S, et al. Distinct phosphorylation sites on the beta(2)-adrenergic receptor establish a barcode that encodes differential functions of beta-arrestin. Sci Signal 2011; 4:ra51.

42 Tiganis T. PTP1B and TCPTP-nonredundant phosphatases in insulin signaling and glucose homeostasis. FEBS J 2013; 280: 445-458.

43 Sarker S, Xiao K, Shenoy SK. A tale of two sites: How ubiquitination of a $\mathrm{G}$ protein-coupled receptor is coupled to its lysosomal trafficking from distinct receptor domains. Commun Integr Biol 2011; 4:528-531.

44 Levkowitz G, Oved S, Klapper LN, et al. c-Cbl is a sup- 
pressor of the neu oncogene. J Biol Chem 2000; 275:3553235539.

45 Marx C, Held JM, Gibson BW, Benz CC. ErbB2 trafficking and degradation associated with $\mathrm{K} 48$ and K63 polyubiquitination. Cancer Res 2010; 70:3709-3717.

46 Eck MJ, Pluskey S, Trub T, Harrison SC, Shoelson SE. Spatial constraints on the recognition of phosphoproteins by the tandem SH2 domains of the phosphatase SH-PTP2. Nature 1996; 379:277-280.

47 Hof P, Pluskey S, Dhe-Paganon S, Eck MJ, Shoelson SE. Crystal structure of the tyrosine phosphatase SHP-2. Cell 1998; 92:441-450.

48 Francis DM, Rozycki B, Koveal D, Hummer G, Page R, Peti W. Structural basis of p38alpha regulation by hematopoietic tyrosine phosphatase. Nat Chem Biol 2011; 7:916-924.

49 Li R, Xie DD, Dong JH, et al. Molecular mechanism of ERK dephosphorylation by striatal-enriched protein tyrosine phosphatase. J Neurochem 2014; 128:315-329

50 Huang Z, Zhou B, Zhang ZY. Molecular determinants of substrate recognition in hematopoietic protein-tyrosine phosphatase. J Biol Chem 2004; 279: 52150-52159.

51 Margottin F, Bour SP, Durand H, et al. A novel human WD protein, h-beta $\operatorname{TrCp}$, that interacts with HIV-1 Vpu connects CD4 to the ER degradation pathway through an F-box motif. Mol Cell 1998; 1:565-574.

52 Menard L, Saadoun D, Isnardi I, et al. The PTPN22 allele encoding an R620W variant interferes with the removal of developing autoreactive B cells in humans. J Clin Invest 2011; 121:3635-3644.

53 Schakman O, Dehoux M, Bouchuari S, et al. Role of IGF-I and the TNFalpha/NF-kappaB pathway in the induction of muscle atrogenes by acute inflammation. Am J Physiol Endocrinol Metab 2012; 303:E729-E739.

54 Pan C, Liu HD, Gong Z, et al. Cadmium is a potent inhibitor of PPM phosphatases and targets the M1 binding site. Sci Rep 2013; 3:2333.

55 Cipolletta D, Feuerer M, Li A, et al. PPAR-gamma is a major driver of the accumulation and phenotype of adipose tissue Treg cells. Nature 2012; 486:549-553.

56 Liang SD, Marmorstein R, Harrison SC, Ptashne M. DNA sequence preferences of GAL4 and PPR1: how a subset of Zn2 Cys6 binuclear cluster proteins recognizes DNA. Mol Cell Biol 1996; 16:3773-3780.

57 Haegens A, Schols AM, Gorissen SH, et al. NF-kappaB activation and polyubiquitin conjugation are required for pulmonary inflammation-induced diaphragm atrophy. Am J Physiol Lung Cell Mol Physiol 2012; 302:L103-L110.

58 van Diepen JA, Vroegrijk IO, Berbee JF, et al. Aspirin reduces hypertriglyceridemia by lowering VLDL-triglyceride production in mice fed a high-fat diet. Am J Physiol Endocrinol Metab 2011; 301:E1099-E1107.

(Supplementary information is linked to the online version of the paper on the Cell Research website.)

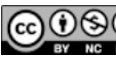
This work is licensed under the Creative Commons Attribution-NonCommercial-No Derivative Works 3.0 Unported License. To view a copy of this license, visit http:// creativecommons.org/licenses/by-nc-nd/3.0 